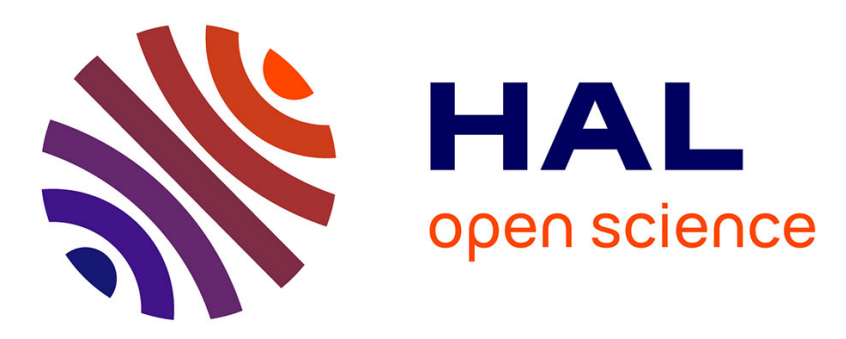

\title{
Synchronisation and control of proliferation in cycling cell population models with age structure
}

Frédérique Billy, Jean Clairambault, Olivier Fercoq, Stéphane Gaubert, Thomas Lepoutre, Thomas Ouillon, Shoko Saito

\section{To cite this version:}

Frédérique Billy, Jean Clairambault, Olivier Fercoq, Stéphane Gaubert, Thomas Lepoutre, et al.. Synchronisation and control of proliferation in cycling cell population models with age structure. Mathematics and Computers in Simulation, 2014, 96, pp.66-94. 10.1016/j.matcom.2012.03.005 . hal00662885

\section{HAL Id: hal-00662885 \\ https://hal.science/hal-00662885}

Submitted on 27 Jan 2012

HAL is a multi-disciplinary open access archive for the deposit and dissemination of scientific research documents, whether they are published or not. The documents may come from teaching and research institutions in France or abroad, or from public or private research centers.
L'archive ouverte pluridisciplinaire $\mathbf{H A L}$, est destinée au dépôt et à la diffusion de documents scientifiques de niveau recherche, publiés ou non, émanant des établissements d'enseignement et de recherche français ou étrangers, des laboratoires publics ou privés. 


\title{
Synchronisation and control of proliferation in cycling cell population models with age structure
}

\author{
Frédérique Billy ${ }^{\mathrm{a}}$, Jean Clairambault ${ }^{\mathrm{a}, *}$, Olivier Fercoq ${ }^{\mathrm{b}}$, Stéphane Gaubert ${ }^{\mathrm{b}}$, \\ Thomas Lepoutre ${ }^{\mathrm{c}}$, Thomas Ouillon ${ }^{\mathrm{d}}$, Shoko Saito ${ }^{\mathrm{e}}$ \\ ${ }^{a}$ INRIA Paris-Rocquencourt \& LJLL, 4 Pl. Jussieu, F75005 Paris, France \\ ${ }^{b}$ INRIA Saclay-Île-de-France \& CMAP, École Polytechnique, 91128 Palaiseau Cedex, France \\ ${ }^{c}$ INRIA Rhône-Alpes \& U. Lyon 1, 43 Bd. du 11 novembre, 69622 Villeurbanne Cedex, France \\ ${ }^{d}$ ENSTA ParisTech, 32 Boulevard Victor, 75739 Paris Cedex 15, France \\ ${ }^{e}$ Department of Genetics, Center for Biomedical Genetics, Erasmus University Medical Center, \\ Rotterdam, The Netherlands; current address: Department of Infection Biology, Faculty of \\ Medicine, University of Tsukuba, Tsukuba, Japan
}

\begin{abstract}
We present and analyse in this article a mathematical question with a biological origin, the theoretical treatment of which may have far-reaching implications in the practical treatment of cancers.

Starting from biological and clinical observations on cancer cells, tumourbearing laboratory rodents, and patients with cancer, we ask from a theoretical biology viewpoint questions that may be transcribed, using physiologically based modelling of cell proliferation dynamics, into mathematical questions.

We then show how recent fluorescence-based image modelling techniques performed at the single cell level in proliferating cell populations allow to identify model parameters and how this may be applied to investigate healthy and cancer cell populations.

Finally, we show how this modelling approach allows us to design original optimisation methods for anticancer therapeutics, in particular chronotherapeutics, by controlling eigenvalues of the differential operators underlying the cell proliferation dynamics, in tumour and in healthy cell populations. We propose a numerical algorithm to implement these principles.
\end{abstract}

Keywords: Mathematical Models, Cell population dynamics, Partial Differential

\footnotetext{
${ }^{*}$ Corresponding author

Email addresses: frederique.billy@inria.fr (Frédérique Billy), jean.clairambaulteinria.fr(Jean Clairambault), olivier.fercoq@inria.fr (Olivier Fercoq), stephane.gaubert@inria.fr (Stéphane Gaubert), thomas. lepoutre@inria.fr(Thomas Lepoutre), thomas.ouillon@gmail.com (Thomas Ouillon), ssaito@md.tsukuba.ac.jp (Shoko Saito)
} 
Equations, Age-structured Models, Cell division cycle, Cancer Drug Toxicity, Chronotherapy, Drug Delivery Optimisation

\section{Contents}

1 Experimental and theoretical motivations 3

1.1 Circadian clocks and tumour growth . . . . . . . . . . . . 3

1.2 Cancer chronotherapeutics $\ldots \ldots \ldots \ldots$

1.3 The goal: chronotherapeutic optimisation in oncology . . . . . . . 6

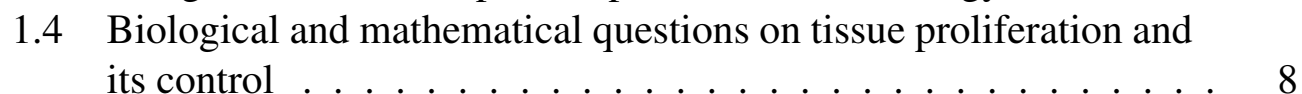

2 Age-structured linear models for tissue proliferation and its control 9

2.1 Basic facts about age-structured linear models . . . . . . . . . 10

2.2 Summary of previous theoretical results . . . . . . . . . . . 11

2.3 New theoretical remarks . . . . . . . . . . . . . . . 12

2.4 Consequences for the cell cycle model . . . . . . . . . . . . . 14

\begin{tabular}{|lll}
3 & Identification of model parameters & 16
\end{tabular}

$3.1 \quad$ FUCCI reporters to identify model parameters . . . . . . . . . . . 16

3.2 Identification procedure . . . . . . . . . . . . . 17

3.3 Numericals $\ldots \ldots \ldots \ldots \ldots \ldots$

3.3.1 Expression of the transition rates . . . . . . . . . . 18

3.3 .2 Discretisation scheme $\ldots \ldots \ldots \ldots . \ldots . \ldots 21$

3.3.3 Numerical determination of the growth exponent . . . . . 22

3.4 Model simulations . . . . . . . . . . . . . 23

3.4 .1 Internal validation . . . . . . . . . . . . . . . . 24

3.4 .2 Numerical simulations . . . . . . . . . . . 24

$4 \quad$ A numerical optimisation problem with toxicity constraints 27

4.1 Prerequisites in nonlinear optimisation . . . . . . . . . . . 27

4.2 Optimisation of the Floquet eigenvalue . . . . . . . . . . . . . . . 29

4.3 Simulations . . . . . . . . . . . . . . . . . 31

5 Discussion and future prospects for this work 35

Submitted July 2011 


\section{Experimental and theoretical motivations}

Tissue proliferation in living organisms always relies on the cell division cycle: one cell becomes two after a sequence of molecular events that is physiologically controlled at each step of the cycle at so-called checkpoints [63, 71]. This process occurs in all renewing tissues, healthy or tumour, but in tumour tissues part of these control mechanisms are inefficient, resulting in uncontrolled tissue growth which may be given as a definition of cancer.

At the initial local stages of cancer (i.e., still without more invasive processes involving tumour neoangiogenesis, digestion of the extracellular matrix and metastases), deficiencies in the control of cell cycle checkpoints, e.g., involving mutated protein $\mathrm{p} 53$, are the main factors responsible for this disrupted control of tissue growth.

The representation of the dynamics of the division cycle in proliferating cell proliferations by physiologically structured partial differential equations (PDEs), which dates back to McKendrick [67], is a natural frame to model proliferation in cell populations, healthy or tumour. Furthermore, the inclusion in such models of targets for its physiological and pharmacological control allows to develop mathematical methods of their analysis and control [28].

\subsection{Circadian clocks and tumour growth}

In the physiological control of the cell division cycle, the role of molecular circadian clocks, which exist in all nucleated cells and are themselves under the control of a central hypothalamic neuronal clock, has been evidenced by numerous animal experiments and is also supported by various clinical observations. These clocks, in the constitution of which about 15 genes have been shown to take part, exert a rhythmic regulating control with a period of approximately 24 hours (hence their name: circa diem = about one day) on hormonal, metabolic, behavioral and proliferative processes [78, 82]. The first of these genes to be discovered were the Per gene in the fruit fly Drosophila Melanogaster by R.J. Konopka and S. Benzer in 1971 [53], and in mammals, the Clock gene by J. Takahashi in 1994 [88]. Their disruption may impair all these physiological processes in an extended manner. It has been experimentally shown by transgenesis experiments that Per2 knock-out transgenic mice are more prone to develop radiation-induced cancers than wild type mice [41].

Similarly, in experiments performed by a totally different team, it has been shown that tumour-bearing mice submitted to artificial strong perturbations of their circadian rhythms constantly exhibit accelerated tumour growth. In a series of articles reporting these experimental observations on two groups of tumourbearing laboratory rodents [39, 40] (the tumour was a fast growing murine tumour positioned in a subcutaneous, easily accessible, site), one with a disrupted central 
circadian clock, the other with a physiologically light-entrained clock as a control group, this has been demonstrated by comparing tumour growth curves. These observations were confirmed and supported by measurements of clock gene expression by quantitative real time polymerase chain reaction (qRTPCR). Furthermore, it was shown that in the disrupted clock group, a partial correction of this tumour growth enhancement was obtained by re-entraining circadian clocks by controlled restricted feeding at fixed and unusual times for rodents (during daylight) [39], thus opening the way to the idea that it could be possible to add an external control to reinforce these physiologically controlled checkpoints when they are deficient.

It has also been observed in clinical settings that patients with cancer, whose circadian rhythms (rest/activity, blood cortisol) were damped or ablated, showed clinically more fatigue and had poorer life prognosis. They also had higher blood levels of cytokines [79], which are emitted by tumour cells, or by immune cells that surround tumours; at least one of these cytokines, $T G F_{\alpha}$, has been experimentally shown, when directly infused in the cerebrospinal fluid of mice (into the third ventricle), to severely disrupt their circadian rhythms, as evidenced on rest-activity and sleep-wake cycles [54].

Whether or not circadian clocks play an essential role in controlling checkpoints of the cell division cycle remains to be more documented, both experimentally, by performing measurements in cell cultures and in living whole organisms, and theoretically, by using and analysing combined mathematical models of molecular circadian clocks and of the cell division cycle in cell populations [28, 31, 32, 33, 35, 36]. Nevertheless, we will develop here the concept of synchronisation between phases of the division cycle in a proliferating cell population, to show that the less synchronised phases are within a cell population (i.e., the looser are cell cycle phase transitions), the faster is proliferation measured by a Malthus-like growth exponent, first eigenvalue of the theoretical tissue growth process.

Furthermore, since circadian clock proteins such as Bmal1 and Per2 have been shown to control cyclin dependent kinases which themselves control in particular $G_{1} / S$ and $G_{2} / M$ phase transitions [65], it is not unlikely from a theoretical biology point of view that circadian clocks control cell cycle phase synchronisation, and by this way, proliferation of the cell population. Consistent with this speculation and the above mentioned experiments on cancer growth enhancement by circadian clock disruption is the fact that in patients with cancer, the less expressed are physiological circadian rhythms, e.g., of rest-activity or blood cortisol, the poorer is the prognosis and the response to cancer treatments [72].

Another speculation that arises from these theoretical considerations is that cancer cell populations might be in this sense, i.e., with respect to cell cycle timing, less synchronised, due or not to circadian clock disruption, than healthy cell 
populations. This remains to be experimentally evidenced, thus by asking a 2stage question: firstly, are cancer cell populations less synchronised than healthy cell ones? and: if so, what is the participation of circadian clock disruption in such desynchronisation? To help better understanding how to experimentally test these hypotheses, we have designed models of the cell division cycle in cell populations and of its periodic control.

\subsection{Cancer chronotherapeutics}

In modern oncology, much before molecular circadian clocks were known, it has been experimentally observed that for anticancer drugs, the time of delivery in the $24 \mathrm{~h}$ span matters. This has led teams of oncologists to use in the clinic of cancers drug delivery schedules with sinusoidal shape implemented in programmable pumps, with $24 \mathrm{~h}$ period and peak delivery times that were determined as the best possible from trials and errors on laboratory animals and subsequently transferred to the clinic [56, 57, 58, 59, 60, 61, 72]. There is a best and a worst time in day for peaks of drug flows to be infused in the general blood circulation, and these peak times depend on the drug used, best times for two different drugs being possibly in antiphase with respect to a $24 \mathrm{~h}$ period. Whence one can deduce that assuming the existence of an optimal cell cycle time with a unique peak drug delivery time, the same for all drugs, is not a correct point of view to guide time-scheduled drug delivery optimisation.

Indeed, these best times depend not only on circadian timing in the cell division cycle (which may be different according to the tissue under consideration, but have been experimentally evidenced in different tissues of living organisms as, e.g., circadian peaks of S-phase, of Cyclin E, of Cyclin B1, etc.) [19, 20, 85], but also on the route followed by drugs in the organism, from their infusion in the general circulation until their action on the DNA in target cells. This last point is the object of pharmacokinetics and it involves drug processing by possibly circadian clock-influenced proteins (hence the name of chronopharmacokinetics) at different levels - blood by binding proteins, liver, target cells - and in particular by intracellular enzymes, which have been shown for some of them to be $24 \mathrm{~h}$ rhythmic, and are different according to the drug under consideration [59].

But most cytotoxic anticancer drugs act in their common final route by inducing damage to the DNA in the nucleus of the cell, which damage is then detected by sensors, such as ATM, that trigger p53 protein, which in turn blocks the cell division cycle, mainly at $G_{1} / S$ or $G_{2} / M$ phase transitions [89, 90, 91]. Others may act directly on phase transition gating, such CDK inhibitors [26].These effects are independent of the chronopharmacokinetic fate of each drug and are the object of molecular pharmacodynamics of cancer, i.e., the discipline that studies 
the molecular effects of drugs on the organisms submitted to anticancer drugs 1

As far as anticancer drugs are concerned, these common terminal molecular effects on the cell division cycle in target cell populations may be represented in cell cycle models, via ATM and p53, or by direct CDK inhibition, by a blockade of the cell cycle at the main checkpoints $G_{1} / S$ and $G_{2} / M[26,64,89,90,91]$, which as mentioned earlier are under the control of circadian clocks, likely in a different manner depending on the nature of the tissue, healthy or tumour. Thus, independently of the drug used (the particularity of which being possibly taken into account by a chronopharmacokinetic model yielding a delay between infusion and presence in cells), it is possible to mathematically represent and optimise the (chronopharmacodynamic) effects of a time-dependent blockade of cell cycle phase transitions in proliferating cell populations, at the terminal level of the DNA in target cells.

Having this in mind, we may tackle the problem of optimising the (hidden) concentration profile in the nucleus of target cells for a generic drug acting by blocking cell cycle transitions. Of course, it should be subsequently completed by a chronopharmacokinetic model for each drug under consideration if one wants to apply results of such optimisation procedures to the clinic. In particular, some drugs act on the DNA all along the cell cycle (e.g., intercalating agents), thus stimulating all checkpoints, while others are specific of $S$ phase (e.g., antimetabolites) or $M$ phase (e.g., spindle poisons), stimulating the time-dependent $G_{2} / M$ checkpoint, or simply blocking mitosis. And it should also be completed by another common model relating DNA damage to p53 triggering (such as [64]). Nevertheless, the terminal chronopharmacodynamic effects on cell cycle checkpoint blockade by assumed time-varying p53 levels can readily be represented as abstract time-dependent mathematical functions to be optimised at the target cell population level.

\subsection{The goal: chronotherapeutic optimisation in oncology}

Optimisation of cancer therapeutics, from a clinical point of view, consists of maximising some therapeutic index of a treatment, measuring (with various definitions) a ratio between its therapeutic outcome in terms of tumour regression and its inevitable toxic side effects on healthy tissues. It thus measures the relevance of the chosen trade-off between therapeutic efficacy and unwanted toxicity. Such an index is hence maximum when there is no toxicity and the tumour is eradicated (a most unlikely situation) and minimum when toxicity is so high that the treatment must be stopped. Giving a mathematical sense to this trade-off is the object of therapeutic optimisation by mathematical methods [1, 2, 3, 9, 27, 29, 30].

\footnotetext{
${ }^{1}$ A useful mnemonic motto with many sources says: "Pharmacokinetics is what the body does to the drug; pharmacodynamics is what the drug does to the body".
} 
Optimising anticancer treatments may plainly consist of defining the drug delivery time schedule (or, rather, its output on p53 or CDK inhibitor concentration at the target cell population level) that will kill as many cancer cells as possible, and then adapt it by trials and errors to clinical tolerability constraints. But since we know that drugs act on proliferating healthy cells (in fast renewing tissues, such as gut epithelium or bone marrow) by the same mechanisms as in cancer drugs, and that these unwanted side-effects on healthy cells are actual clinical issues that limit the use of these drugs, it is legitimate to consider the drug delivery optimisation problem as a problem of optimisation under constraints, the solution of which is a trade-off between the objective function (decreasing the cancer cell population) and the constraint (maintaining the healthy cell population over a tolerable level).

The constraint mentioned here is a simple constraint consisting of limiting the drug delivery output at the cell level (p53 or CDK inhibitor concentration) in a given healthy cell population in such a way that the cell population level will absolutely remain, whatever the circumstances, over a predefined limit threshold, e.g., $50 \%$ of an assumed equilibrium cell density level. Other constraints have clear clinical meanings, but are more difficult to represent: a) drug resistance in cancer cells, that can be drug dose-dependent and leads to limit the administration of a given drug, for fear of developing resistant cancer cell clones [43, 52], a problem also encountered e.g., in antibiotherapy; b) drug toxicity to immune cells which fight cancer locally, and may be affected by deleterious effects of drugs [92], to be thus represented as indistinct artillery fire over two fighting cell populations, the good and the bad one; c) drug toxicity to circadian clocks [60, 61] that can perturb their assumed synchronising effects on the cell division cycle. All these supplementary constraints, realistic though they are, have not yet been taken into account in models, partly by lack of biological knowledge of these toxicity mechanisms. We will limit ourselves to the representation of the parallel action of the same drug on two different cell populations, a cancer one and a healthy one, without any communication between them, hence simultaneously a therapeutic effect and unwanted toxic side effects.

It is also possible to take into account individual parameters of the cell population models, simple or more complex, based on their identification in groups of patients defined by common responses to drugs, genetically or epigenetically determined, opening the way to personalised medicine. This level of representation takes place at the level of populations of patients, not of cells, and relies on statistical populational pharmacokinetic-pharmacodynamic (PK-PD) models. Although it should certainly be considered in the future, starting from a molecular point of view, it is concerned with levels that are presently way downstream of the cell molecular level that we will describe here by differential equations to describe cell population dynamics at the tissue level. 
Placing ourselves within the limited modelling frame of a generic drug acting in parallel on a healthy tissue and on a tumour, both represented by cell population dynamic systems of equations, it has already been shown that it was possible, using a simplified set of ordinary differential equations (ODEs) with a physiological circadian clock control on the pharmacodynamics of a one-drug external control, to obtain optimised drug delivery schedules, solutions to an optimisation problem under constraints [9, 27]. These schedules are optimal in the sense that, constraining the healthy cell population to remain over an absolute tolerability threshold (absolute, but adaptable to what could be the patient's state of health), the tumour cell kill is maximised by a delivery drug flow that takes into account the effects of circadian clocks on both cell populations.

The assumption used there was that there was a best time to kill tumour cells, which was at the same time the best to preserve healthy cells from toxicity. Although it has found some experimental support explored in [9, 27], this assumption may be forsaken to comply with the more likely conjecture of a clear obedience of healthy proliferating cells to circadian clock synchronising messages, and a looser obedience to, or total ignorance of, the same messages in tumour cells. It is this assumption that we will now put forward, as it has also been used by others in different modelling settings [1, 2, 3].

\subsection{Biological and mathematical questions on tissue proliferation and its control}

The biological theoretical questions arising from these observations made in laboratory experimental and clinical conditions have been partially sketched above or in previously published papers; all of them still remain open. They deal mainly with a possible connection between periodic (circadian) control of proliferation and tumour growth rate, with therapeutic consequences in oncology. It has been shown in particular [31, 32, 33, 35, 36] that there is no natural hierarchy between growth exponents compared according to periodic control vs. no control on cell cycle phase transition rates.

Here, we ask another, more precise, type of question, focusing, as mentioned above, on age synchronisation within the cell cycle.

- At the cell population level with control on phase transitions, what are the differences between healthy and cancer cells? A speculation, mentioned above, proposes that healthy cells are well synchronised within the division cycle in the cell population (roughly speaking, and caricaturing, all cells in phase $i$ pass to phase $i+1$ of the division cycle at the same age) while cancer cells would show more variability with respect to age at transition, i.e., are desynchronised, or at least less synchronised than healthy cells. Is this true, can it be observed in cell cultures or in living animals and how? And what is the experimentally observed effect of a better synchronisation on proliferation speed? 
- To connect this speculation with observations on circadian clock-controlled proliferating cell populations, a corresponding speculation is that circadian clocks enhance synchronisation with respect to cell cycle timing in proliferating cell populations. Is this true, can it be experimentally observed and how?

- It has been mentioned above that measurable effects of chronotherapeutics are most likely due to both chronopharmacokinetics of drug processing cell molecules and to physiological circadian control of checkpoints at cell cycle phase transitions. Is it possible to decouple these effects and directly study the control on the cell cycle, independently of the drug used?

These biological questions have corresponding items in mathematical terms when one uses the McKendrick model [67] paradigm for proliferating cell population dynamics. They can be investigated by analytical or numerical methods. They read:

- Excluding in the first place time-dependent control by circadian clocks and focusing on cell cycle phase transitions dependent only on age (corresponding e.g., to populations of proliferating cells in cell cultures without any external synchronisation signal), what may be said about the influence on the first eigenvalue - which governs the long-time behaviour of the proliferating cell population as a growth (or Malthus) exponent [76], see Section 2 - of a desynchronisation between cells at a phase transition?

- Now, if one adds time-dependent, and periodic, control on these phase transitions, what more can be said? Is it possible to correct by time-dependent control the effects of desynchronisation of cells with respect to age in the cell cycle?

- In particular, assuming that cancer cell populations are less synchronised than healthy ones, how can we represent both physiological circadian control (and its possible disruptions) and theoretical therapeutical control to design optimised cancer chronotherapeutics at the cell cycle level?

We will in the following sections state remarks, towards answering these questions, that may help a new understanding of the biological and mathematical problem, and finally we leave pending open questions for the discussion.

\section{Age-structured linear models for tissue proliferation and its control}

Physiologically structured cell population dynamics models have been extensively studied in the last 25 years, see $e . g$. [4, 5, 6, 68]. We consider here typically 
age-structured cell cycle models. If we consider that the cell division cycle is divided into $I$ phases (classically 4: $G_{1}, S, G_{2}$ and $M$ ), we follow the evolution of the densities $n_{i}(t, x)$ of cells having age $x$ at time $t$ in phase $i$. Equations read :

$$
\left\{\begin{array}{l}
\frac{\partial n_{i}(t, x)}{\partial t}+\frac{\partial n_{i}(t, x)}{\partial x}+d_{i}(t, x) n_{i}(t, x)+K_{i \rightarrow i+1}(t, x) n_{i}(t, x)=0, \\
n_{i+1}(t, 0)=\int_{0}^{\infty} K_{i \rightarrow i+1}(t, x) n_{i}(t, x) d x, \\
n_{1}(t, 0)=2 \int_{0}^{\infty} K_{I \rightarrow 1}(t, x) n_{I}(t, x) d x .
\end{array}\right.
$$

Together with an initial condition $\left(n_{i}(t=0, .)\right)_{1 \leq i \leq I}$. This model was first introduced in [34]. The particular case $I=1$ has received particular attention from the authors [31, 32]. In this model, in each phase, the cells are ageing with constant speed 1 (transport term), they may die (with rate $d_{i}$ ) or go to next phase (with rate $K_{i \rightarrow i+1}$ ) in which they start with age 0 . We write it in its highest generality. If we want to represent the effect of circadian rhythms, we usually consider time-periodic coefficients, the period being of course $24 h$.

Note that we will not consider here a resting phase $\left(G_{0}\right)$, with exchanges with the $G_{1}$ phase. It is certainly possible to do it in a biologically more realistic setting, since even in fast renewing tissues, all cells are not in in a proliferative state [24, 87], and also since it has been done already [10,11, 14], even in a completely linear setting [42] (i.e., without nonlinear feedback). Nevertheless, since the observations on the basis of which we performed the identification of our model parameters (see below Section 3 report recordings on proliferating cells only, we have limited our theoretical frame here to the classical $G_{1}, S, G_{2}$ and $M$ phases.

\subsection{Basic facts about age-structured linear models}

One of the most important facts about linear models is its trend to exponential growth. Solutions to (1) satisfy (if the coefficients are time-periodic, or stationary) $n_{i}(t, x) \sim C^{0} N_{i}(t, x) e^{\lambda t}$, where $N_{i}$ are defined by (for $T$-periodic coefficients)

$$
\left\{\begin{array}{l}
\frac{\partial N_{i}(t, x)}{\partial t}+\frac{\partial N_{i}(t, x)}{\partial x}+\left(\lambda+d_{i}(t, x)+K_{i \rightarrow i+1}(t, x)\right) N_{i}(t, x)=0, \\
N_{i+1}(t, 0)=\int_{0}^{\infty} K_{i \rightarrow i+1}(t, x) N_{i}(t, x) d x \\
N_{1}(t, 0)=2 \int_{0}^{\infty} K_{I \rightarrow 1}(t, x) N_{I}(t, x) d x \\
N_{i}>0, \quad N_{i}(t+T, .)=N_{i}(t, .), \quad \sum_{i} \int_{0}^{T} \int_{0}^{\infty} N_{i}(t, x) d x d t=1 .
\end{array}\right.
$$

The study of the growth exponent $\lambda$, first eigenvalue of the system, thus governs the long-time behaviour of the population (since the $N_{i}$ are bounded) and is therefore of crucial importance. For further reading about the asymptotic behaviour of (1), the reader may consult [76] (chapter 3) for an overview of the subject. 
In the 1-phase case $(I=1)$, i.e., if only the total cell cycle duration is taken into consideration, extended studies of the first and second eigenvalues (there is only one positive eigenvalues, but others exist, that are complex) of the system have been performed in [25], following [68], with proposed experimental methods using flow cytometry to identify these eigenvalues. In the present study, we will not consider other eigenvalues than the first one, but we are fully aware of the fact that considering the second eigenvalue (its real and imaginary parts) may be of importance if one wants to precisely describe in particular transient phenomena that appear when control changes occur at cell cycle phase transitions.

\subsection{Summary of previous theoretical results}

Sharp study of the properties of the growth exponent $\lambda$ has been done, based on previous studies [69, 70] by some of the authors in [31, 32, 33, 35, 36] where the dependency of the first eigenvalue on the control of the death rates $d_{i}(t, x)$ or of the phase transition rates $K_{i \rightarrow i+1}(t, x)$ by a periodic function was the main object of investigation.

In this part, we focus on the case of stationary phase transition coefficients $\left(K_{i \rightarrow i+1}(t, x)=K_{i \rightarrow i+1}(x)\right)$ and we do not consider death rates. As shown in [34], the first eigenvalue $\lambda$ is then given as the only positive solution to the following equation, which in population dynamics is referred to, in the 1-phase case $(I=1)$ with no death term, as Lotka's (or Euler-Lotka) equation:

$$
\frac{1}{2}=\prod_{i=1}^{I} \int_{0}^{+\infty} K_{i \rightarrow i+1}(x) e^{-\int_{0}^{x} K_{i \rightarrow i+1}(\xi) d \xi} e^{-\lambda x} d x
$$

It can be seen by straightforward computation (see below Section 2.4), in this case with stationary coefficients, that $\lambda$ is a nondecreasing function of the $K_{i \rightarrow i+1}$ (and it is worth noticing that this is no longer the case when the coefficients depend on age and time, as depicted in [55]). We also recall that, integrating equation (1) along its characteristics, we can in the stationary case with no death rate derive the formula:

$$
n_{i}(t+x, x)=n_{i}(t, 0) e^{-\int_{0}^{x} K_{i \rightarrow i+1}(\xi) d \xi} .
$$

This can be interpreted in the following form: the probability that a cell which entered phase $i$ at time $t$ stays for at least an age duration $x$ in phase $i$ is given by

$$
P\left(\tau_{i} \geq x\right)=e^{-\int_{0}^{x} K_{i \rightarrow i+1}(\xi) d \xi} .
$$

This leads to the natural consideration of the time $\tau_{i}$ spent in phase $i$ as a random variable on $\left[0,+\infty\left[\right.\right.$, with probability density function $\varphi_{i}$ :

$$
d P_{\tau_{i}}(x)=\varphi_{i}(x) d x=K_{i \rightarrow i+1}(x) \cdot e^{-\int_{0}^{x} K_{i \rightarrow i+1}(\xi) d \xi} d x
$$


Notice that it is necessary for this interpretation to be coherent, and $\varphi_{i}$ to be a probability density function, to impose $\int_{0}^{+\infty} K_{i \rightarrow i+1}(x) d x=+\infty$, which physiologically means that all cells leave any phase in finite time, without any hypothetical maximum age in phase to be introduced in the cell cycle model. In the sequel we will naturally use the notation $E\left[e^{-\lambda \tau}\right]=\int_{0}^{+\infty} e^{-\lambda x} d P_{\tau}(x)$. In particular, the classical (1-phase) case in Lotka's equation then reads $E\left[e^{-\lambda \tau}\right]=\frac{1}{2}$.

\subsection{New theoretical remarks}

\section{Order structure on random variables.}

Definition 2.1. The stochastic ordering is defined in the following way: for two real-valued random variables $X, Y$, we say that $X \leq{ }^{\text {st }} Y$ if for all $t \in \mathbb{R}$,

$$
P(X>t) \leq P(Y>t) .
$$

The following application is then straightforward:

Proposition 2.2. The growth rate is non increasing with respect to stochastic ordering.

Note that the growth rate reverses stochastic order on cell cycle durations because the higher in this order is actually the slower to lead to division (although it would be false to think that mean cell cycle time would exactly give mean doubling time, which is by definition, asymptotically $\tau_{d}=\ln 2 / \lambda$; see $e$.g. from a statistical estimation point of view [86], where it is proposed that if $C C T$ is the random variable "cell division cycle time", then $\tau_{d}=E[C C T]-\frac{1}{2} \lambda \operatorname{Var}[C C T]+o(\lambda)$ for small values of $\lambda$ ). But equivalently, one may state that order on growth rate respects order on cumulative distribution functions.

Proof. We show it in the 1-phase case $I=1$ (it extends immediately to the general case from Lotka's equation (3) above by considering stochastic orders in each phase $i$ separately). This is based on classical integration by parts: let $\lambda>0$

$$
E\left[e^{-\lambda X}\right]=\int_{0}^{\infty} e^{-\lambda t} P_{X}(d t)=\lambda \int_{0}^{\infty} e^{-\lambda t} P(X \leq t) d t .
$$

Therefore for any $\lambda>0$,

$$
E\left[e^{-\lambda X}-e^{-\lambda Y}\right]=\lambda \int_{0}^{\infty} e^{-\lambda t}\{P(Y>t)-P(X>t)\} d t \geq 0 \quad \text { if } X \leq^{s t} Y .
$$

Whence, using Lotka's equation for both $X$ and $Y$,

$\int_{0}^{\infty} e^{-\lambda(Y) \cdot t} P_{Y}(d t)=E\left[e^{-\lambda(Y) \cdot Y}\right]=E\left[e^{-\lambda(X) \cdot X}\right] \geq E\left[e^{-\lambda(X) \cdot Y}\right]=\int_{0}^{\infty} e^{-\lambda(X) \cdot t} P_{Y}(d t)$,

therefore $\lambda(X) \geq \lambda(Y)$ if $X \leq^{s t} Y$. 
Dispersion enhances proliferation. Another useful concept coming from the field of probability is the concept of dilation. One says that a measure $\mu$ is a dilation of $\nu$ if for any convex function $\phi$, one has

$$
\int \phi(x) \mu(d x) \geq \int \phi(x) \nu(d x)
$$

We have the following

Proposition 2.3. Suppose $\mu$ is a dilation of $\nu$, then $\lambda(\mu) \geq \lambda(\nu)$.

Proof. Since $\phi(x)=e^{-\lambda(\nu) x}$ is convex, we have by definition

$$
\int e^{-\lambda(\nu) x} \mu(d x) \geq \frac{1}{2}
$$

and therefore $\lambda(\mu) \geq \lambda(\nu)$.

Two examples. It is worth giving an important example of dilation.

Let $X$ be a random variable of law $\mu$, let $X_{\theta}=E[X]+\theta(X-E[X])$, with correponding law $\mu_{\theta}$, for $\theta>0$. Then the following holds

$$
\left\{\begin{array}{l}
\text { If } \theta<1, \quad \mu \text { is a dilation of } \mu_{\theta}, \\
\text { If } \theta>1, \quad \mu_{\theta} \text { is a dilation of } \mu .
\end{array}\right.
$$

This means that increasing the dispersion around the mean increases the proliferation (if expectation is fixed).

We now prove this fact for $\theta<1$ (the other case is then an immediate consequence of the fact that $X=\left(X_{\theta}\right)_{\frac{1}{\theta}}$. Let $\phi$ be a convex function. Then

$\int \phi(x) d \mu_{\theta}(x)=E[\phi((1-\theta) E[X]+\theta X)] \leq(1-\theta) \phi(E[X])+\theta E[\phi(X)] \leq E[\phi(X)]$

(using Jensen's inequality).

Another example is the following: let $\left(X_{i}\right)_{0 \leq i \leq n}$ be a sequence of i.i.d. random variables. For $\alpha_{i} \geq 0, \sum_{i} \alpha_{i}=1$, we denote $Y^{\alpha}=\sum_{i=1}^{n} \alpha_{i} X_{i}$ and $\mu^{\alpha}$ the law of $Y^{\alpha}$, we recall then the definition of majorization for vectors:

Definition 2.4. Let $\alpha, \beta \in \mathbb{R}_{+}^{n}$ with $\sum \alpha_{i}=\sum \beta_{i}=1$. Denote $\alpha^{\downarrow}, \beta^{\downarrow}$ the decreasing rearrangements of $\alpha, \beta$, we say that $\alpha$ weakly majorizes $\beta$ (denoted $\alpha \succ_{w} \beta$ ) if we have for all $j \leq n$,

$$
\sum_{i \leq j} \alpha_{i}^{\downarrow} \geq \sum_{i \leq j} \beta_{i}^{\downarrow}
$$

or equivalently, if there exists of a doubly stochastic matrix $P$ such that $\beta=P \alpha$. 
We then have the following

Proposition 2.5. Suppose $\alpha \succ_{w} \beta$, then $\mu^{\alpha}$ is a dilation of $\mu^{\beta}$.

Proof. Using the above notation, and $X=\left(X_{1}, \ldots X_{n}\right)$ we know that

$$
Y^{\beta}=\beta \cdot X^{T}=\alpha P X^{T}
$$

Recall that from the Birkhoff theorem, we have

$$
P=\sum_{\sigma \in S_{n}} \lambda_{\sigma} P^{\sigma}, \lambda_{\sigma} \geq 0, \quad \sum \lambda_{\sigma}=1
$$

where $P^{\sigma}$ are the permutation matrices. Therefore we have

$$
Y^{\beta}=\sum \lambda^{\sigma} \alpha P^{\sigma} X^{T}
$$

For a convex function $\phi$, we then have

$E\left[\phi\left(Y^{\beta}\right)\right] \leq \sum \lambda_{\sigma} E\left[\phi\left(\alpha P^{\sigma} X^{T}\right)\right]=\sum \lambda_{\sigma} E\left[\phi\left(\alpha X^{T}\right)\right]=E\left[\phi\left(\alpha X^{T}\right)\right]=E\left[\phi\left(Y^{\alpha}\right)\right]$.

In particular, an elementary consequence of this remark is that it recovers the fact that the growth exponent associated to a single random variable is higher than the one associated to the mean of $n$ i.i.d. representatives of the same random variable. Furthermore, it indicates a hierarchy in a panel of intermediate situations between these two extreme situations, $(0,0, \ldots, 0,1)$ and $\left(\frac{1}{n}, \frac{1}{n}, \ldots, \frac{1}{n}\right)$ for convex combinations of i.i.d. random variables and the resulting probability laws, a hierarchy that is relevant for comparing growth exponents.

\subsection{Consequences for the cell cycle model}

First, as noted above, it is straightforward (as for Proposition (2.2), using Lotka's equation) to see that in the case of the cell division cycle model with stationary coefficients $K_{i \rightarrow i+1}(x)$ and no death rates, where

$$
P(X>x)=e^{-\int_{0}^{x} K_{X}(\xi) d \xi},
$$

the order $K_{X} \leq K_{Y}$ implies $\lambda(X) \leq \lambda(Y)$. In fact it may be shown that this is also here a definition of hazard rate order - reversing orders on random variables: $X \geq_{h r} Y$-, which in our case is equivalent to stochastic order. One may conclude in general that the looser (in the sense $K_{X} \geq K_{Y}$ ) is the control on the transition coefficients $K_{i \rightarrow i+1}(x)$, or equivalently the higher is the cumulative distribution function $1-e^{-\int_{0}^{x} K_{X}(\xi) d \xi}$, the higher the growth exponent $\lambda$.

Second, the first example of dilation, consequence of Proposition (2.2), above admits the following easily understandable consequence: 
Let a cell division cycle model with no death rates and with stationary phase transition coefficients $\mathrm{K}_{i \rightarrow i+1}(x)$ be given by its probability density functions

$$
\varphi_{i}(x)=K_{i \rightarrow i+1}(x) \cdot e^{-\int_{0}^{x} K_{i \rightarrow i+1}(\xi) d \xi}, 1 \leq i \leq I .
$$

Assume that for a given phase $i$, there exists a number $\theta>1$ such that the random variable $X_{i}$, duration in age of phase $i$, takes its values in $\left[\frac{\theta-1}{\theta} E\left[X_{i}\right],+\infty[\right.$. This is in particular possible, as shown by elementary calculus, for probability density functions of random variables on $\mathbb{R}_{+}$of the Gamma type

$$
\beta^{\alpha}(\Gamma(\alpha))^{-1}(x-\gamma)^{\alpha-1} e^{-\beta(x-\gamma)} \mathbb{1}_{[\gamma ;+\infty[}(x),
$$

where $\gamma>0$ and $\mathbb{1}_{[\gamma ;+\infty[}$ is the indicator function of interval $[\gamma ;+\infty[$. To the probability density function $\varphi_{i}$ for age duration $X_{i}$ in phase $i$ corresponds $\varphi_{i}^{\theta}$, probability density function of $Y_{i}^{\theta}=X_{i}+\theta\left(X_{i}-E\left[X_{i}\right]\right)$. Then $Y_{i}^{\theta} \geq 0$, and if $\theta>1$, $\varphi_{i}^{\theta}$ is a dilation of $\varphi_{i}$, with $E\left[Y_{i}^{\theta}\right]=E[X]$ and $\operatorname{Var}\left[Y_{i}^{\theta}\right]=\theta^{2} \operatorname{Var}[X]>\operatorname{Var}[X]$. Hence $\lambda\left(Y_{i}^{\theta}\right)>\lambda\left(X_{i}\right)$, thus providing us with an example of dilation, relying on an increase in dispersion, which results in an enhancement of the growth exponent $\lambda$.

Another example is (again) the Gamma distribution $\beta^{\alpha}(\Gamma(\alpha))^{-1} x^{\alpha-1} e^{-\beta x}$ on $\mathbb{R}_{+}$in which we fix the expectation $E[X]=\mu=\frac{\alpha}{\beta}$; then $\operatorname{Var}[X]=\frac{\mu}{\beta}$, and a straightforward calculation using Lotka's equation (3) in the 1-phase case yields

$$
\lambda=\beta\left(2\left(\frac{1}{\mu \beta}\right)-1\right) .
$$

Again, elementary calculus shows that this expression is always decreasing with $\beta$, i.e., increasing with $\operatorname{Var}[X]$, when the mean duration $\mu$ of the phase or of the total division cycle is kept constant.

Third, an example of the most elementary consequence of Proposition 2.5 may be illustrated again by using the Gamma distribution, with constant expectation $\mu$ and size parameter $\alpha=k$ integer (then it is also known as Erlang distribution and it is the distribution of the arithmetic mean of $k$ i.i.d. random variables with exponential distribution of scale parameter $\beta=\frac{k}{\mu}$ ). Here

$$
\lambda(k)=k\left(2^{\left(\frac{1}{k}\right)}-1\right)
$$


an expression that decreases (towards $\ln 2$ ) when $k$ increases, i.e., when the variance $\frac{\mu^{2}}{k}$ of the distribution decreases.

Note that we have given here examples of probability laws with fixed expectation for which increasing the variance results in enhancement of $\lambda$. The choice of the Gamma distribution has not been chosen haphazardly, as the next section will show. Now, how far does the growth exponent $\lambda$ depend on the variance of the duration of the same phase (or of the total cycle duration) in a cell cycle model in general? It can be seen that if $X$ and $Y$ do not belong to the same family of probability laws, $\operatorname{Var}[X] \leq \operatorname{Var}[Y]$, together with $E[X]=E[Y]$, does not imply $\lambda(X) \leq \lambda(Y)$ in general. But as shown above, if $X$ and $Y$ do belong to the same family, which is for instance the case for the Gamma distribution family, that will be of high interest for us in the sequel, this is true.

\section{Identification of model parameters}

Other authors [84] have used comparable modelling to investigate the cell cycle in cell populations, but the novelty of our contribution in this section is that we have used recent image data on individual cells that enable us to assess the variability of cell cycle phase durations in populations of cells.

\subsection{FUCCI reporters to identify model parameters}

From a biological point of view, the cell cycle is classically considered as composed of 4 phases named $G_{1}$ (gap 1), $S$ (DNA synthesis), $G_{2}$ (gap 2) and $M$ (mitosis). One challenge of our modelling study was to determine the expression of the parameters $d_{i}$ and $K_{i \rightarrow i+1}$ mentioned in the model (1) for each phase of the cell cycle $(i=1 \ldots 4)$. We concentrated our efforts on $K_{i \rightarrow i+1}$, assuming that $d_{i}=0$ for all $i=1 \ldots 4$. Note that if one assumes a constant death rate $d$ for all phases, then it is included (negatively) in the growth exponent $\lambda$, which in fact may be thought of as the minimal added artificial death rate that stabilises the cell population, as results from equations (2).

To get an expression for these transition rates, we needed to have access to the distribution of the duration of the phases of the cell cycle within a cell population.

FUCCI is the acronym of fluorescent ubiquitination-based cell cycle indicator. This is a recently developed technique that allows tracking progression within the cell cycle of an individual cell with a high degree of contrast [80, 81]. The FUCCI method consists in developing two fluorescent probes indicating whether a tracked cell is in the $G_{1}$ phase or in one of the phases $S, G_{2}$ or $M$ of the cell cycle. The authors fused red- and green-emitting fluorescent proteins to proteins called Cdt1 and Geminin. Cdt1 and Geminin oscillate reciprocally: Cdt1 level is highest in 
the $G_{1}$ phase and falls down when the cell enters the $S$ phase, whereas Geminin level is highest in the $S, G_{2}$ and $M$ phases and falls when the cell enters the $G_{1}$ phase. Let us mention that Cdt 1 and Geminin are degraded due to the process of ubiquitination, which is what is referred to (" $U$ ") in the name of the reporter method. Consequently, the nucleus of a FUCCI cell fluoresces in red when this cell is in the phase $G_{1}$, and in green when it is in $S, G_{2}$ or $M$ phases.

This method allows to measure the time a tracked cell spends in the $G_{1}$ phase and the remaining part $S / G_{2} / M$ of the cell cycle. By tracking each cell in a population (note that cell tracking is not a completely trivial imagery problem because in liquid media cells move) we can get the distributions of the duration of these phases within the population, and so we can deduce the probability density functions of the random variables representing the duration of these phases (see below Subsection 3.3 for details).

\subsection{Identification procedure}

We used for the parameter identification procedure FUCCI data transmitted to us within the C5Sys EU project by G. van der Horst's team, Erasmus University Medical Center, Rotterdam, The Netherlands. The cell lines were obtained by one of us (S. Saito at Erasmus University) by recloning cell cycle phase markers (in the proper colour combination, see further) and generating/analysing NIH 3T3 cells (mouse embryonic fibroblasts) proliferating in a liquid medium. The data processed in the identification procedure thus consisted of time series of intensities recording the red and green fluorescences emitted by individual NIH 3 T3 cells proliferating within an in vitro homogeneous population. These cells had not been preliminarily synchronised, which means that they were initially at different stages of the cell cycle. The intensities had been recorded every fifteen minutes, over approximately 38 hours. A graph representing such a time series is presented on Figure 1 .

We considered in the mass of data (about 2000 tracked cells) that were available to us only those (about 50) with at least the duration of a complete cell cycle, and measured the duration of the $G_{1}$ and $S / G_{2} / M$ phases within this cell cycle (note here that since all the cells that were kept for parameter identification were alive from the beginning of the experiment until its end, the assumption of a zero death rate in the model is in full accordance with these particular experimental conditions). The end of a cell cycle is characterised by a fast disappearance of the green fluorescence, so that it was not difficult to measure the duration of the cell cycle on our data. During the transition from $G_{1}$ to $S$, red and green fluorescences overlap, so that it is not so easy to determine the duration of phase $G_{1}$. In agreement with our biologist partners, we decided to define the end of phase $G_{1}$ as the time at which red fluorescence was maximum before decreasing. The duration of 


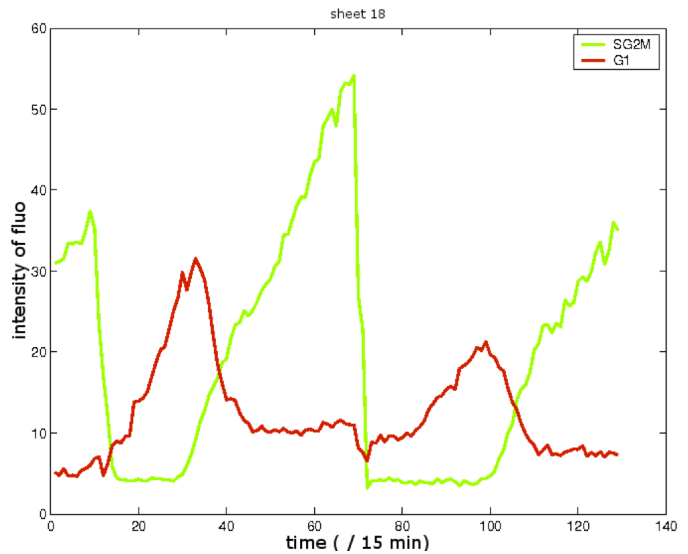

Figure 1: Example of a time series of the intensity of red (deep grey) and green (light grey) fluorescences obtained by using the FUCCI method on a NIH 3T3 cell within a population in liquid medium.

phase $S / G_{2} / M$ was obtained by subtracting the duration of phase $G_{1}$ from the duration of the cell cycle. This method is summarised on Figure 2.

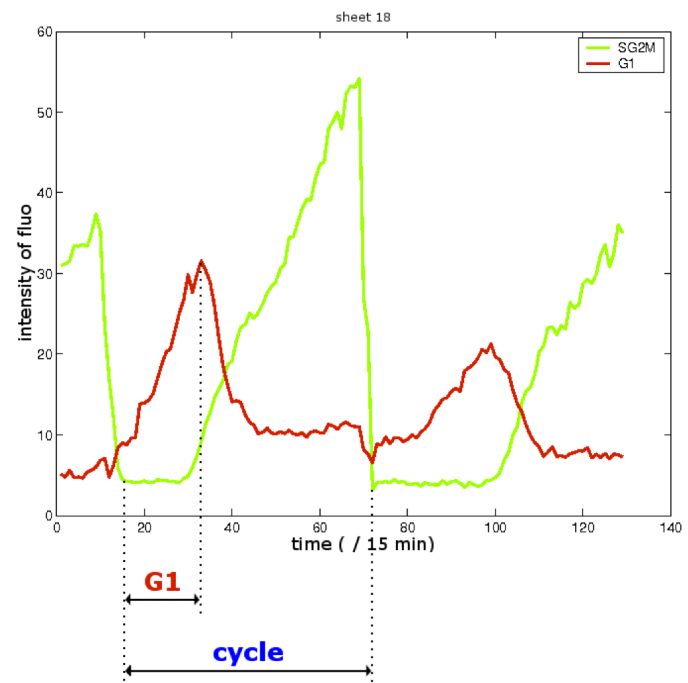

Figure 2: Graphic method used to determine the duration of the cell cycle and the one of $G_{1}$ phase. The duration of phase $S / G_{2} / M$ was deduced by subtracting the duration of phase $G_{1}$ from the duration of the cell cycle.

\subsection{Numericals}

\subsubsection{Expression of the transition rates}

Using this identification method, we obtained 55 data on individual cells for the duration of the cell cycle, divided in $G_{1}$ and $S / G_{2} / M$ phases. The mean value 
of the duration of the cell cycle was about $17.1 \mathrm{~h}$ (s.d.: $4.5 \mathrm{~h}$ ), the one of $G_{1}$ was about $7.2 \mathrm{~h}$ (s.d.: $2.7 \mathrm{~h}$ ), and thus the one of $S / G_{2} / M$ was about $9.8 \mathrm{~h}$ (s.d.: 3.0 h).

We rounded each duration to the nearest hour. The distributions of the durations of $G_{1}$ and of $S / G_{2} / M$ within the population were fitted to experimental data by using Gamma laws. The corresponding curves are presented on Figure 3 . We tested several models to fit experimental data. We excluded laws that had support not strictly contained in $\mathbb{R}_{+}$, such as normal laws; similarly, we excluded laws that assumed a maximum age in phase, which is impossible to define naturally and furthermore results in difficulties when identifying their parameters. We chose Gamma laws because they allowed a good (phenomenological) fit to our experimental data while keeping a reasonable number of parameters to be estimated.

Moreover, there is a clear physiological basis to this choice of the Gamma distribution: recall that, if the parameter $\alpha$ is an integer, it is the law, often used to represent probabilities of waiting times of the sum of $\alpha$ i.i.d. random variables representing waiting times (here within $G_{1}$ or $S / G_{2} / M$ of times between triggerings of crucial switches in a cascade of protein expressions leading to a phase transition, e.g., $G_{1} / S$ ), each one of them following an exponential law with the same parameter $\beta$. Such an explanation, or parts of it, has been proposed in this context or others dealing with gene or protein expression by many authors, let us only mention [37, 66, 83]. Note that here the Gamma distribution is not used, as in [14] and references therein, to represent the distribution of maturation times for cells performing a fixed number of divisions, but the distribution of times spent in a phase of the cell division cycle, supposed to be constituted of a cascade of (unidentified, hidden physiological) switches, as mentioned above, hence its as phenomenological as physiological justification in our case.

For all $x \geq 0$, we thus used the following probability density functions, where $\Gamma$ is the Gamma function:

$$
\begin{gathered}
\varphi_{i}(x)=\frac{1}{\Gamma\left(\alpha_{i}\right)}\left(x-\gamma_{i}\right)^{\alpha_{i}-1} \beta_{i}^{\alpha_{i}} e^{-\beta_{i}\left(x-\gamma_{i}\right)} \mathbb{1}_{\left[\gamma_{i} ;+\infty[\right.}(x) \quad i=1,2, \quad \text { where } \\
\alpha_{1}=8.28, \beta_{1}=1.052 h^{-1}, \gamma_{1}=0 h, \alpha_{2}=3.42, \beta_{2}=1.47 h^{-1}, \gamma_{2}=7.75 h
\end{gathered}
$$

and $\mathbb{1}_{\left[\gamma_{i} ;+\infty[\right.}$ is the indicator function of interval $\left[\gamma_{i} ;+\infty[\right.$. These parameters led to a mean duration and a standard deviation on $\mathbb{R}_{+}$respectively of $7.9 \mathrm{~h}$ and $2.7 \mathrm{~h}$ for the $G_{1}$ phase and of $10.1 h$ and $1.3 h$ for the $S / G_{2} / M$ phase. These figures are very close to the ones related to the raw experimental data mentioned above. The main difference resides in the $S / G_{2} / M$ data and is due to the fact that we have identified a high position parameter $\left(\gamma_{2}=7.75 h\right)$ for the Gamma distribution in $S / G_{2} / M$, which may be interpreted as an "incompressible" minimum duration for the $S / G_{2} / M$ part of the cell division cycle in our observed cell population, hence, en passant, an indirect measure of this cell physiological parameter, which 
is the minimum age a cell has to spend in $S / G_{2} / M$ before being able to process further and divide. Similarly, the figure 8.28 for $\alpha_{1}$, compared to 3.42 for $\alpha_{2}$ may be interpreted - a speculation- as due to the presence of many more biological switches (and resulting stopping times) in $G_{1}$ than in $S / G_{2} / M$.
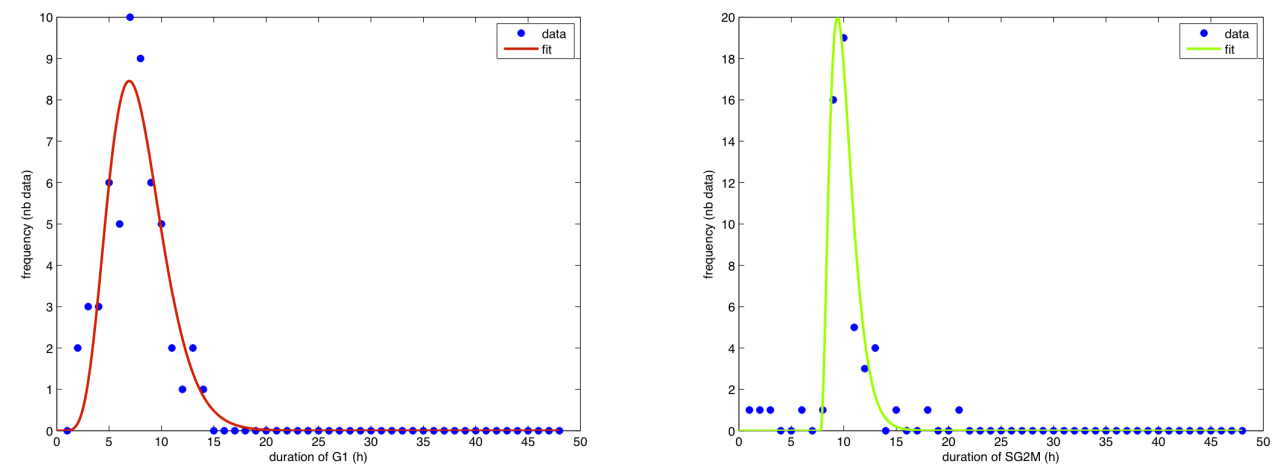

Figure 3: Gamma laws (solid line) (multiplied by a coefficient equal to the total number of data, i.e. 55) that fit experimental data (dots) for the distribution of the duration of $G_{1}$ (left) and of $S / G_{2} / M$ (right).

Let us also mention that in a close, but different context (linear age- structured population, one proliferation phase and presence of a death term), others have proposed an inverse problem method to identify, without any assumption on a probabilistic model for the cell cycle time, the birth and death functions [48].

Let us now come back to the 2-phase mathematical model ( $I=2$ in model (1)).

As the experimental data were performed in vitro in a liquid medium, with no intercellular communication, and as cells had not been synchronised prior to the experiment, we can consider that there was no time-dependent control whatsoever on the growth process at the cell population level. We thus assumed that the transition rates from $G_{1}$ to $S / G_{2} / M\left(K_{1 \rightarrow 2}\right)$ and from $S / G_{2} / M$ to $G_{1}\left(K_{2 \rightarrow 1}\right)$ did not depend on time, but only on the age of cells in the two phases. From the expression of the cumulative distribution function mentioned in Section 2 .

$$
\int_{0}^{x} \varphi_{i}(\xi) d \xi=1-e^{-\int_{0}^{x} K_{i \rightarrow i+1}(\xi) d \xi} \quad i=1,2
$$

we deduce:

$$
K_{i \rightarrow i+1}(x)=\frac{\varphi_{i}(x)}{1-\int_{0}^{x} \varphi_{i}(\xi) d \xi} \quad i=1,2
$$

where $\varphi_{i}$ represents the experimentally determined probability density function of the random variable representing the age duration in phase $i$. One may note here 
that the right hand side is known in general as the hazard rate that has been briefly mentioned in Subsection (2.4).

The graphs of the transition rates we obtained from formula (5) and experimental data are presented on Figure 4.
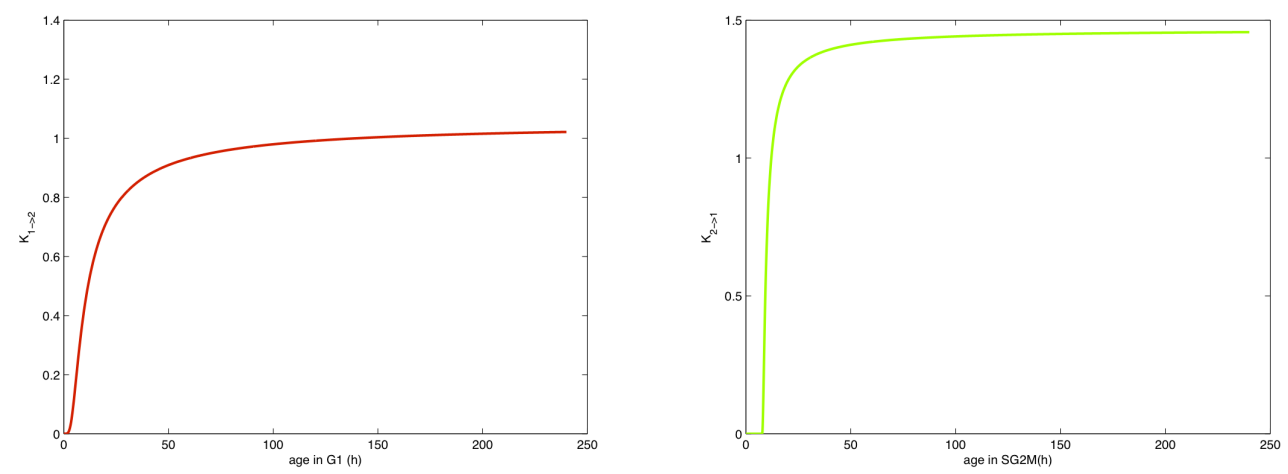

Figure 4: Transition rates from $G_{1}$ to $S / G_{2} / M$ (left) and from $S / G_{2} / M$ to $G_{1}$ (right). These rates are functions of age of cells in the phases only.

\subsubsection{Discretisation scheme}

In this part, we come back to the general case where $K_{i \rightarrow i+1}$ depends on age and time. Thus far, we had always considered that $d_{i}=0$ but, for numerical convenience, we also had supposed that cells could not grow beyond a maximal age. Numerically assessing this assumption, we came to the conclusion that it had no influence on the value of $\lambda>0$ provided that this maximal age was large enough, so that, for numerical convenience, we arbitrarily took a maximal age of 10 days for each one of the 2 phases.

We base our discretisation scheme on the one presented in [31]. We denote by $\Delta t$ and $\Delta x$ respectively the time and age steps, i.e. $t=k \Delta t$ and $x=j \Delta x$ where $k=0 \ldots\left\lfloor\frac{T_{t o t}}{\Delta t}\right\rfloor$ and $j=0 \ldots\left\lfloor\frac{X_{t o t}}{\Delta x}\right\rfloor,\lfloor z\rfloor$ representing the integer part of the real number z, and $T_{t o t}$ and $X_{t o t}$ being respectively the total time of the simulation and the maximal time a cell can spend in one phase of the cell cycle. For $k \in \mathbb{N}, j \in \mathbb{N}$ and for $i=1,2$, we consider the following quantities:

$$
\begin{gathered}
n_{i}^{k, j}=n_{i}(k \Delta t, j \Delta x) \\
K_{i \rightarrow i+1}^{k, j}=K_{i \rightarrow i+1}(k \Delta t, j \Delta x)
\end{gathered}
$$

We use a first order finite difference scheme on the 2-phase mathematical 
model. Assuming $\Delta t=\Delta x(\mathrm{CFL}=1)$, we have:

$$
\begin{array}{rlrl}
n_{i}^{k+1, j} & =\frac{n_{i}^{k, j-1}}{1+\Delta t K_{i \rightarrow i+1}^{k+1, j}} & i & =1,2, k=0 \ldots k_{\max }-1, j=1 \ldots j_{\max } \\
n_{i}^{0, j} & =n_{i}^{0}(j \Delta x) & i & =1,2, j=0 \ldots j_{\max } \\
n_{1}^{k+1,0} & =2 \sum_{j=0}^{j_{\max }} \frac{\Delta t K_{2 \rightarrow 1}^{k+1, j+1}}{1+\Delta t K_{2 \rightarrow 1}^{k+1, j+1}} n_{2}^{k, j} & & k=0 \ldots k_{\max }-1 \\
n_{2}^{k+1,0} & =\sum_{j=0}^{j_{\max }} \frac{\Delta t K_{1 \rightarrow 2}^{k+1, j+1}}{1+\Delta t K_{1 \rightarrow 2}^{k+1, j+1}} n_{1}^{k, j} & k & =0 \ldots k_{\max }-1
\end{array}
$$

where $k_{\text {max }}=\left\lfloor\frac{T_{\text {tot }}}{\Delta t}\right\rfloor, j_{\text {max }}=\left\lfloor\frac{X_{\text {tot }}}{\Delta x}\right\rfloor, n_{i}^{0}$ denoted the initial density of cells in each of the two phases.

Unlike the scheme presented in [31], this scheme ensures mass conservation of cells through the phase transitions for any discretisation step. Indeed we have the relation

$$
\sum_{j \geq 0} n_{1}^{k, j}=n_{2}^{k+1,0}+\sum_{j>0} n_{1}^{k+1, j}+\frac{n_{1}^{k, j_{\max }}}{1+\Delta t K_{1 \rightarrow 2}^{k+1, j_{\max }+1}}
$$

which means that cells at time $k$ either go to next phase or get older or go beyond the age limit and are thus "killed." This property gives a better coherence with biological reality.

\subsubsection{Numerical determination of the growth exponent}

If transition rates do not depend on time, which is the case when there is no circadian control, the first eigenvalue $\lambda$ of the system (2) is given by equation (3). In the particular case of our experimentally based study described above, we have:

$$
\left(1+\frac{\lambda}{\beta_{1}}\right)^{\alpha_{1}}\left(1+\frac{\lambda}{\beta_{2}}\right)^{\alpha_{2}} e^{\lambda\left(\gamma_{1}+\gamma_{2}\right)}=2
$$

In our experimental case, with the coefficients of two Gamma distributions identified from FUCCI data (see above), this yields $\lambda \approx 0.039 h^{-1}$.

In the general case, transition rates depend on time. Insofar as we are interested in the effect of circadian rhythms on cell cycle progression, we restrict this study to time-periodic transition rates, the period being equal to $T=24$ hours. Over a time period, let $n^{k}=\left(n_{1}^{k, 0}, n_{1}^{k, 1}, \ldots, n_{1}^{k, j_{\max }}, n_{2}^{k, 0}, \ldots, n_{2}^{k, j_{\max }}\right)$ be a vector in $\mathbb{R}^{2\left(j_{\max }+1\right)}$. As in the case of the 1-phase model presented in [31], we can write 
$n^{k+1}=M_{k} n^{k}$ where $M_{k}=\left(\begin{array}{ll}A 1_{k} & B 2_{k} \\ B 1_{k} & A 2_{k}\end{array}\right)$ where $A i_{k}$ and $B i_{k}$ are $\left(j_{\max }+1\right)^{2}$ matrices defined by:

$$
A i_{k}=\left(\begin{array}{ccccc}
0 & \cdots & \cdots & 0 & 0 \\
\frac{1}{1+\Delta t K_{i \rightarrow i+1}^{k+1,1}} & & & \vdots & \vdots \\
0 & \ddots & & \vdots & \vdots \\
\vdots & & \ddots & 0 & 0 \\
0 & \cdots & 0 & \frac{1}{1+\Delta t K_{i \rightarrow i+1}^{k+1, j \max }} & 0
\end{array}\right) \quad i=1,2
$$

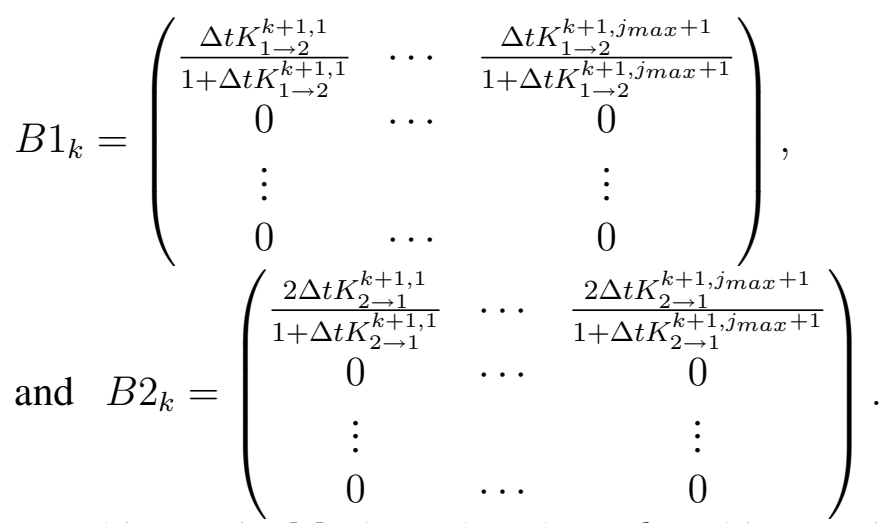

This matrix $M_{k}$ depends only on $k$ and is T-periodic. Moreover, if we define the matrix $\mathbb{M}=M_{N_{T}} M_{N_{T}-1} \ldots M_{2} M_{1}$ where $N_{T}=T / \Delta t$, we have $n^{N_{T}}=\mathbb{M} n^{0}$. The following proposition is simple adaptation of its analogue in [31].

Proposition 3.1. If for all phases $i$ and for all $t \in\left[0 ; T_{\text {tot }}\right]$, there exists $x_{0} \in$ $\left[0 ; X_{\text {tot }}\right]$ such that $K_{i \rightarrow i+1}(x, t)>0$ for all $x \geq x_{0}$, then $\mathbb{M}=M_{N_{T}} M_{N_{T}-1} \ldots M_{2} M_{1}$ is a nonnegative and irreducible matrix.

By the Perron-Frobenius theorem (see [12] for instance), this proposition means that $\mathbb{M}$ has a simple and positive principal eigenvalue $\rho$ associated with nonnegative left and right eigenvectors unique up to a given normalisation. We calculated this eigenvalue $\rho$ by means of the power algorithm [46]. The Floquet eigenvalue, or growth exponent, $\lambda$ can then be approximated by $\frac{1}{T} \log (\rho)$.

\subsection{Model simulations}

As we are interested in the role of circadian rhythms on cell cycle progression, we consider transition rates of the form : $K_{i \rightarrow i+1}(x, t)=\kappa_{i}(x) \cdot \psi_{i}(t) \quad(i=1,2)$, where $\kappa_{i}$ corresponds to the transition rates we identified in Subsection 3.3, and $\psi_{i}$ represents a $24 \mathrm{~h}$-periodic time control exerted by circadian rhythms on cell cycle progression at phase transitions. 


\subsubsection{Internal validation}

To make sure that our numerical results were in agreement with the biological data that we used to build our model ("internal validation"), we performed simulations in the case of no time control, that is $K_{i \rightarrow i+1}(x, t)=\kappa_{i}(x)(i=1,2)$, where the $\kappa_{i}(x)$ were given by the expression in (5) $\left(\kappa_{i}(x)=K_{i \rightarrow i+1}(x)\right)$. Figure 5 presents the time evolution of the percentage of cells in phases $G_{1}$ and $S / G_{2} / M$ over the duration of one cell cycle resulting from numerical and biological experiments (biological data were preliminarily synchronised "by hand", i.e., by deciding that all cells were at age nought at the beginning of simulations). We had to reduce this comparison to the duration of one cell cycle because we had not enough biological data to represent the whole population. We can nevertheless notice that modelled numerical data were very close to raw biological data. We are thus entitled to conclude that the model and the method we have used to represent the proliferation phenomenon and fit our experimental data may have led us close to biological likelihood.

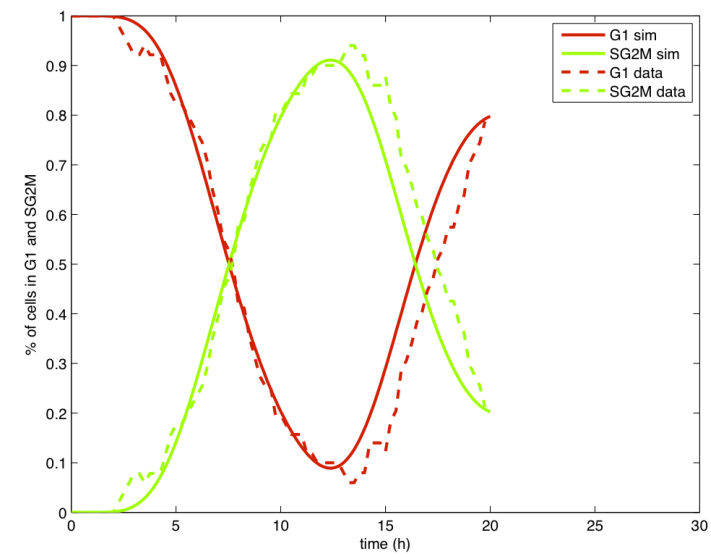

Figure 5: Time evolution of the percentages of cells in $G_{1}$ (red or deep grey) and $S / G_{2} / M$ (green or light grey) phases from biological data (dashed line) and from numerical simulations (solid line). Our model results in a good approximation of the biological data.

\subsubsection{Numerical simulations}

In the case of no time control $\left(\psi_{i} \equiv 1, i=1,2\right)$, we studied the time evolution of the percentages of cells in $G_{1}$ and $S / G_{2} / M$ phases of the cell cycle and the evolution of the total density of cells. These results are presented on Figure 6 . We can notice that oscillations are damped and that the percentages rapidly reach a steady state. This phenomenon, which has long been known in cell population dynamics [4, 5, 6, 25, 68] as asynchronous growth, is the result of desynchronisation of cells through the cell cycle: although the cells were taken initially all in 
phase $G_{1}$ with age 0 , the variability in the duration of phases $G_{1}$ and $S / G_{2} / M$ we described above induces some variability in the position of cells through their cycling. In our experimental case, as mentioned above, the exponential growth exponent $\lambda$ computed by using Lotka's equation (3) was equal to $0.039 h^{-1}$.
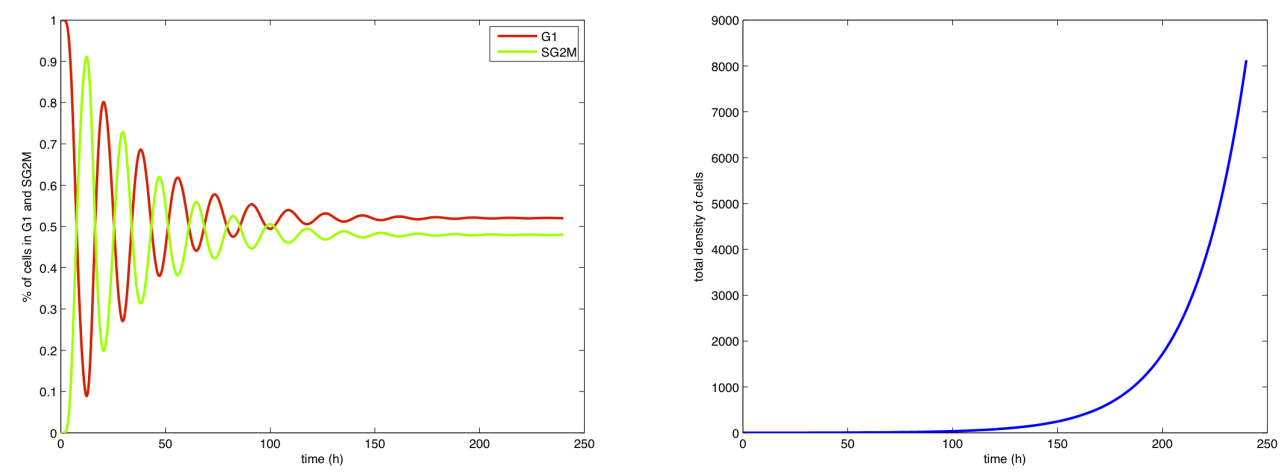

Figure 6: Time evolution of the percentages of cells $G_{1}$ (left, red or deep grey) and $S / G_{2} / M$ (left, green or light grey) phases and of the total density of cells (right) evolving free of any time control. Oscillations are damped due to cell desynchronisation. Population growth tends to be exponential with a rate equal to $0.039 h^{-1}$.

Then, we introduced a circadian control modelled by functions $\psi_{i}(i=1,2)$ in the transition rates. For each phase, $G_{1}$ and $S / G_{2} / M$ we took continuous piecewise 24h-periodic cosine functions, represented on Figure 7, defined on [0;24] by:

$\psi_{1}(t)=\cos ^{2}(2 \pi(t-3) / 12) \mathbb{1}_{[12 ; 18]}(t)+\varepsilon, \quad \psi_{2}(t)=\cos ^{2}(2 \pi(t-3) / 12) \mathbb{1}_{[0 ; 6]}(t)+\varepsilon$, where $\varepsilon=10^{-10}$ ensures $\psi_{i}>0$ (which may be shown sufficient to imply irreducibility of matrix $\mathbb{M}$ and thus applicability of the Perron-Frobenius theorem).

These phenomenological cosine-like functions standing here for physiological circadian control on cell cycle phase transitions in fact represent the local impact of the central circadian clock control onto the cell division cycle. This impact may be thought of either as retransmitted by a direct action of glucocorticoids - known to be synthesized on a circadian basis by the corticosurrenal gland [23] - directly on the $G_{1} / S$ transition via stimulation of p27 and inhibition of cMyc [7, 8], or as retransmitted by another relay involving local circadian clocks and control by Bmal1 of the complex Cyclin B-Cdk1 that controls the $G_{2} / M$ transition [65]. In the absence of an actual experimental identification of the main gating variables (Cyclin E-Cdk2 on $G_{1} / S$ and Cyclin B-Cdk1 on $G_{2} / M$ ) and the circadian control on them, we have chosen to represent them by such truncated cosines.

The $12 \mathrm{~h}$ delay between the definition of $\psi_{1}$ and $\psi_{2}$ is suggested by biological observations, that teach us that circadian controls are exerted on the main check- 
points $G_{1} / S$ and $G_{2} / M$ and that proteins $p 21$ and $W e e 1$, known circadian controls on these checkpoints, are expressed in antiphase [47]. Furthermore, and completely independently of such physiological knowledge, we remarked by varying the phase delay that this value of 12 hours maximised the population growth exponent $\lambda$. We thus obtained periodic transition rates $K_{i \rightarrow i+1}(x, t)=\psi_{i}(t) . \kappa_{i}(x)$ of mixed origin, estimated by cosines $\left(\psi_{i}(t)\right)$ and experimentally determined $\left(\kappa_{i}(x)\right)$.
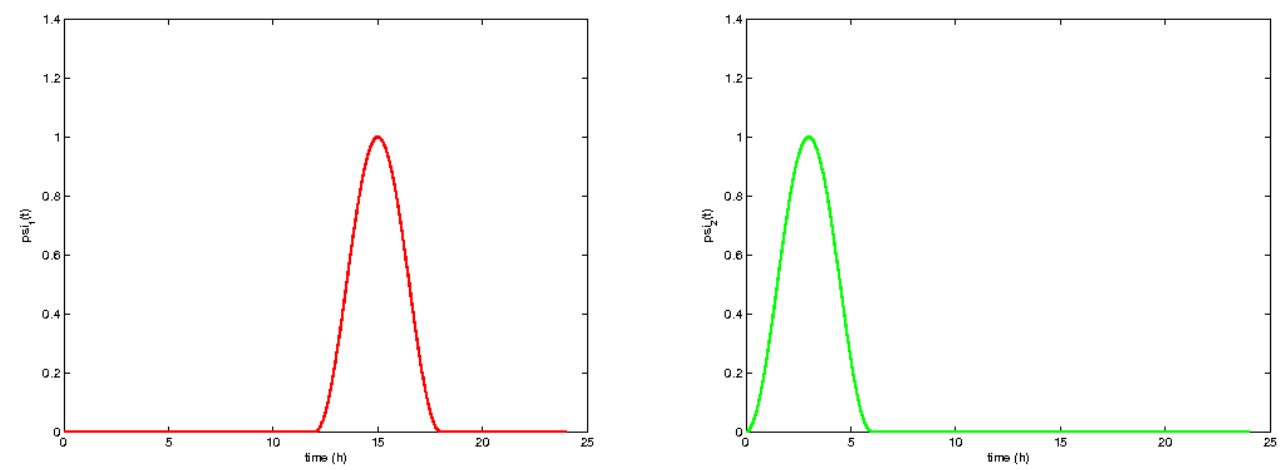

Figure 7: Graphic definition of the 24h-periodic functions $\psi_{1}$ (left) and $\psi_{2}$ (right) modelling circadian control on the cell cycle. $\psi_{1}$ controls $G_{1}$ to $S$ transition, and $\psi_{2}$ controls $M$ to $G_{1}$ transition.

In this case, we observed that oscillations of the percentages of cells in $G_{1}$ and in $S / G_{2} / M$ were no more damped (see Figure 8 ). In fact, because of circadian entrainment, cells were more synchronised in the cell cycle and consequently divided approximately all together. Moreover, the exponential growth rate was about $0.024 h^{-1}$, which means that the population dynamics was slower than the one without time control we investigated above. Thus, in this case, circadian rhythms allowed cells to be more synchronised and to divide slower.

These first numerical simulations tend to be in agreement with the biological hypothesis according to which populations of cells that can escape circadian control, such as cancer cell population could do, proliferate faster than populations of cells submitted to circadian entrainment.

As shown in [31, 32, 33, 35, 36], there cannot be a general theoretical result for periodic control on cell cycle transitions, comparing proliferation in controlled and uncontrolled situations. Nevertheless, it could be that the particular form of the periodic control investigated here, i.e., the product of an age-dependent component which may be qualified as hazard rate $\kappa_{i}(x)$ of a Gamma distribution for phase duration, and of a periodic time-dependent component $\psi_{i}(t)$ with optimised phase shift between the two transition control functions $\psi_{1}$ and $\psi_{2}$, does result in slowing down the cell cycle speed. This remains to be both experimentally and theoretically investigated. 

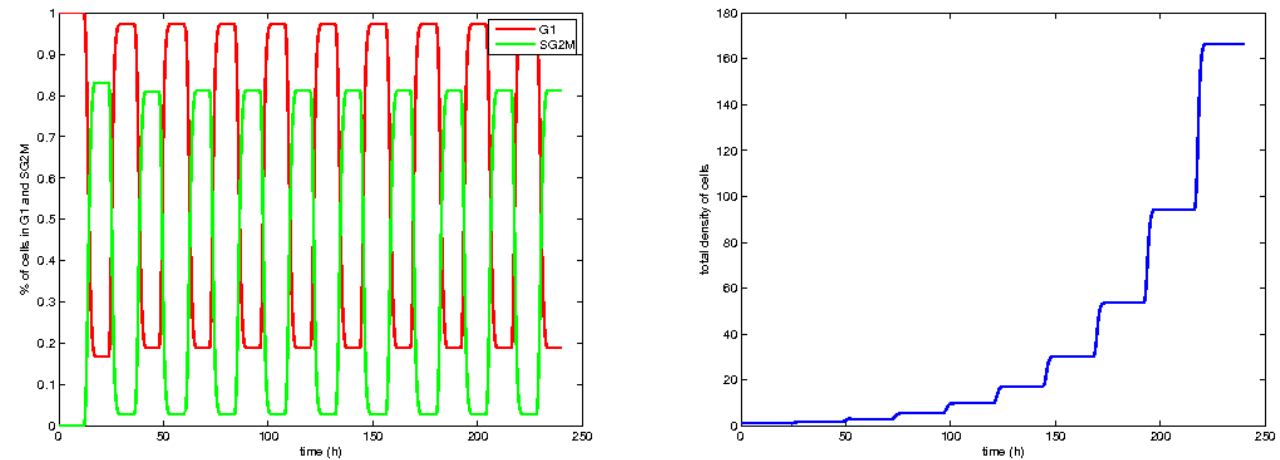

Figure 8: Time evolution of the percentages of cells in phases $G_{1}$ (left, red or deep grey) and $S / G_{2} / M$ (left, green or light grey) and of the total density of cells (right) submitted to circadian control. Oscillations are not damped any more. Population growth tends to be exponential with a rate equal to $0.024 h^{-1}$.

\section{A numerical optimisation problem with toxicity constraints}

We study in this section the problem of minimising the growth rate of cancer cells subject to a toxicity constraint on healthy cells. A related problem is studied in [9]. As in that paper, we consider two populations called healthy and cancer cells that do not interact but are subject to circadian control and drug infusion. The populations behave differently and the goal is to find a drug infusion scheme that attacks cancer cells more than healthy cells.

Our approach is however different here because we consider an age-structured model with several phases for the population dynamics. Moreover, whereas the authors of [9] consider an initial distribution of cells and the consequence of the drug on this initial distribution, we consider asymptotic growth rates. We thus describe long-range drug treatment in a stationary state by controlling growth exponents, i.e., first eigenvalues of both cell population systems, healthy and cancer, simultaneously.

\subsection{Prerequisites in nonlinear optimisation}

An optimisation problem with implicit constraints can be written in the following general form:

$$
\begin{array}{r}
\min _{x \in X} F_{0}(x) \\
F_{i}(x)=0, \quad i \in I \\
F_{i}(x) \leq 0, \quad i \in J
\end{array}
$$

The set $X \subset \mathbb{R}^{n}$ represents constraints that are easily tractable, like for instance bound constraints. The function $F_{0}: \mathbb{R}^{n} \rightarrow \mathbb{R}$ is the objective function or cost 
function and the functions $F_{i}: \mathbb{R}^{n} \rightarrow \mathbb{R}, i \neq 0$, define implicit constraints: $\left\{F_{i}(x)=0\right\}$ is an equality constraint, $\left\{F_{i}(x) \leq 0\right\}$ is an inequality constraint. We assume here that for all $i \in\{0\} \cup I \cup J$, the function $F_{i}$ is differentiable.

An important particular case is when there are no implicit constraints. The optimisation problem becomes then:

$$
\min _{x \in X} F_{0}(x)
$$

There exists a vast literature on the resolution of such problems [22, 17, 73] but we will concentrate on first order methods, i.e. optimisation algorithms that only need the value of the function and its first derivative. Indeed, as we study the discretisation of a continuous problem, we can deal with a huge number of variables. Computing and storing the Hessian matrix of a function can be very time-consuming when it has many variables, so even when we have an explicit formula, we do not use it.

The simplest first order optimisation algorithm for Problem $(8)$ is the projected gradient method [44, 45]. It requires the set $X$ to be convex and uses the orthogonal projection $P_{X}$ on $X$. Starting from $x_{0}$, the algorithm builds the sequence $\left(x_{k}\right)_{k \geq 0}$ such that:

$$
x_{k+1}=P_{X}\left(x_{k}-\alpha_{k} \nabla F_{0}\left(x_{k}\right)\right)
$$

The step size $\alpha_{k}$ can be either fixed or determined dynamically. A simple generalisation of this algorithm is to replace $\nabla F_{0}\left(x_{k}\right)$ by another vector $d_{k}$ called the descent direction.

For a general nonconvex optimisation problem, one cannot guarantee that the optimum has been reached. Instead, one considers stationary points, where a point $x^{*}$ is said to be stationary if

$$
\left\langle\nabla F\left(x^{*}\right), x^{*}-x\right\rangle:=\sum_{i=1}^{n} \frac{\partial F}{\partial x_{i}}\left(x^{*}\right)\left(x_{i}^{*}-x_{i}\right) \leq 0, \quad x \in X .
$$

Proposition 4.1 ([45]). If $\nabla F_{0}$ is Lipschitz with constant $L$ and $0<\alpha<\frac{2}{L}$, then every limit point of the projected gradient algorithm with fixed step $\alpha$ is a stationary point of (8).

One can also free oneself of the Lipschitz constant assumption by performing a line search for the determination of the length step $\alpha_{k}$. A simple line search rule is the Armijo step-size rule on the projected arc [15]. We fix $\sigma \in] 0,1[, \beta \in] 0,1[$ and $s>0$. Then $\alpha_{k}=\beta^{m} s$ where $m$ is the first nonnegative integer such that

$$
F\left(x_{k}\right)-F\left(x_{k}\left(\beta^{m} s\right)\right) \geq \sigma \frac{\| x_{k}-x_{k}\left(\beta^{m} s \|^{2}\right.}{\beta^{m} s} .
$$


When we have implicit constraints, we use the method of multipliers for the resolution of Problem (7). This method is described precisely in [16].

Given $c>0$, we call augmented Lagrangian of the Problem (7) the function $L_{c}$ defined by

$$
L_{c}(x, \mu)=F(x)+\sum_{j \in I}\left(\mu_{j} F_{j}(x)+\frac{c}{2} F_{j}(x)^{2}\right)+\frac{1}{2 c} \sum_{j \in J}^{K}\left(\max \left(0, \mu_{j}+c F_{j}(x)\right)^{2}-\mu_{j}^{2}\right)
$$

The method of multipliers consists in the following scheme, starting with $\mu^{0}$ :

$$
\begin{aligned}
x^{k} & =\arg \min _{x} L_{c_{k}}\left(x, \mu^{k}\right) \\
\mu_{j}^{k+1} & =\mu_{j}^{k}+c_{k} F_{j}\left(x^{k}\right), \quad j \in I \\
\mu_{j}^{k+1} & =\max \left(0, \mu_{j}^{k}+c_{k} F_{j}\left(x^{k}\right)\right), \quad j \in J
\end{aligned}
$$

where the minimisation is understood to be local in a ball within which $x$ is the unique local minimum of Problem (8). Under classical assumptions (see [16] for instance), the method of multipliers converges to a stationary point of the constrained optimisation Problem (7).

\subsection{Optimisation of the Floquet eigenvalue}

We study the problem of optimising the first eigenvalue with control in a set of matrices. That is, we study the optimisation of the growth rate in the discretised model.

Unlike in [31, 32], we will refer to the Perron eigenvalue when we consider nonnegative square matrices and to the Floquet eigenvalue when we study the growth exponent of a time-periodic controlled population. Indeed, if we denote by $\rho$ the Perron eigenvalue of the matrix $M$ defined by the discretisation scheme in Section 3.3.3, which means that $M u=\rho u$ and $\rho$ has maximal modulus, then the Floquet eigenvalue, $\lambda$ can then be approximated by $\frac{1}{T} \log (\rho)$.

By the Perron-Frobenius theorem [12], we know that if $M$ is nonnegative and irreducible, its principal eigenvalue $\rho(M)$ is positive and is a simple eigenvalue. Moreover, as mentioned above, the principal eigenvector is unique up to a normalisation and can be chosen such that $u(M) \geq 0$. In our setting (irreducibility of the matrix and the Perron-Frobenius theorem), one can naturally define a function $\rho$ from the open set of nonnegative and irreducible real matrices in $\mathbb{R}^{n}$ into $\mathbb{R}_{+}$, that to a matrix associates its principal eigenvalue.

General eigenvalue optimisation of non symmetric matrices is a difficult (non convex, non differentiable) problem: see [62] and [75] for two algorithms dealing with this problem. However, for positive matrices, as the principal eigenvalue is simple, this implies that $\rho$ is differentiable: 
Proposition 4.2 ([50]). Denoting $v$ and $u$ the left and right eigenvectors of a matrix $M$ associated to a simple eigenvalue $\rho$, the derivative of $\rho$ at $M$ can be written as:

$$
\frac{\partial \rho}{\partial M_{i j}}=v_{i} u_{j}
$$

Thus, as the objective function is differentiable, the theory of differentiable optimisation applies (see Section 4.1).

As stressed by Overton in [74], there are various possibilities for the computation of the eigenvalue and eigenvectors. Here, we consider sparse nonnegative matrices with a simple principal eigenvalue: the power method applies and, unlike direct methods or inverse iterations, it only needs matrix-vector products, which is valuable with a large sparse matrix.

The Perron (or Floquet) eigenvalue optimisation problem with explicit constraints can be written as:

$$
\min _{M \in h(C)} f(\rho(M))
$$

We assume that $f$ is a real-valued twice continuously differentiable function; $C$ a compact convex set and we denote $P_{C}$ the orthogonal projection on $C ; h$ is a twice continuously differentiable function such that $\mathcal{M}=h(C)$ is a set of nonnegative irreducible matrices.

We may also need implicit constraints on the eigenvalues. The Perron (or Floquet) eigenvalue optimisation problem with $K=|I|+|J|$ implicit constraints can be written as:

$$
\begin{array}{r}
\min _{x \in C} f_{0}\left(\rho\left(h_{0}(x)\right)\right) \\
f_{k}\left(\rho\left(h_{k}(x)\right)\right)=0, \quad k \in I \\
f_{k}\left(\rho\left(h_{k}(x)\right)\right) \leq 0, \quad k \in J
\end{array}
$$

To solve this non convex problem, we use the method of multipliers [16], which solves a sequence of non constrained optimisation problems $(10)$ whose solutions converges to the solution of the constrained problem (11).

For our application, $x$ will be the array of the infusion time step by time step and drug by drug. The various functions $h_{i}$, one by type of cell considered (healthy or cancer, bone cell or intestine cell...), represent the dependence of the model upon drug infusion. We may also set implicit constraints on $x$ directly.

The objective could be for instance the maximisation of the growth rate (for healthy cells) with $f_{0}(r)=-\frac{1}{T} \log (r)$, the minimisation of the growth rate (for cancer cells) $f_{0}(r)=\frac{1}{T} \log (r)$. For the constraints, we may consider a lower bound $\Lambda$ (toxicity threshold) for the growth rate of healthy cells by $f_{1}(r)=$ $-\frac{1}{T} \log (r)+\Lambda$ or a prescribed total daily dose $D$ by $f_{2}(x)=\sum_{t} x_{t}-D$. 


\subsection{Simulations}

We propose numerical simulations for the problem of a drug infusion strategy that minimises the growth rate of cancer cells $\left(f_{0}(r)=-\frac{1}{T} \log (r)\right)$ while maintaining the growth rate of healthy cells above a threshold $\Lambda=0.022 h^{-1}$ $\left(f_{1}(r)=-\frac{1}{T} \log (r)+\Lambda \leq 0\right)$.

Infusion here may be thought of as referring to the drug 5-Fluorouracil (5FU), an anticancer drug with $S$ phase specificity, acting via DNA damage and involving a preserved p53 protein control (having nevertheless in mind that p53 is mutated in many cancers [49, 89]); but it may also be thought of, in all cases of cancer, as referring to the delivery of a CDK inhibiting molecule (CDKI) - some of which have shown antimitotic activity [26] - , thus directly blocking $G_{1} / S$ or $G_{2} / M$ transitions, or both. The pharmacological impact of the drug is thus represented by an added phase transition blockade, as illustrated on Figures 9 and 10 .

In Section 3 , we have determined the parameters of the cell population dynamics model with two phases $G_{1}$ and $S / G_{2} / M$. We thus consider two phases in our simulations. We chose a discretisation step of 6 minutes because it may be considered as a lower limit to the half-life time of 5-FU in the plasma [21, 38, 77], which is most likely even lower than the half-time of its downstream molecular effects at the cell level, our concern here. The oldest ages represented in the discretisation scheme are 10 days for each phase.

Transitions from one phase to the other are described by the transition rates $K_{i \rightarrow i+1}(t, x)$. We take them with the form $K_{i \rightarrow i+1}(t, x)=\kappa_{i}(x) \psi_{i}(t)\left(1-g_{i}(t)\right)$ where $\kappa_{i}(x)$ is the transition rate of the cell without circadian control identified in Section 3, $\psi_{i}(t)$ is the natural circadian control and $g_{i}(t)$ is the effect at the cell level of the drug infusion at time $t$ on the transition rate from phase $i$ to phase $i+1$. No drug corresponds to $g_{i}(t)=0$, a transition-blocking infusion corresponds to $g_{i}(t)=1$. If the modelled drug is 5-FU, it acts on phase $S$ (and thus on the aggregated phase $S / G_{2} / M$ ) on the DNA, resulting in damaged DNA and subsequent blocking control at the $G_{2} / M$ transition only; we then have $g_{1}(t)=0$ for all $t$, and $g_{2}$ only is controlled. Numerical experiments suggest that optimal drug infusion patterns are 1-day periodic, so we restrict to 1-day periodic drug infusion strategies.

We considered two cell populations called cancer cells and healthy cells. In these simulations, we made them differ only by the circadian time controls $\psi_{i}$ between cell cycle phases $i$ and $i+1$, and we assumed that there was no interaction between the two populations, healthy and cancer.

We took for this circadian control a continuous truncated piecewise cosine function (i.e., $\cos ^{2}$ times an indicatrix function) for each phase. For healthy cells, we took the functions described in Section 3.4, that is, we located the circadian control around 3 a.m. for the transtion from $S / G_{2} / M$ to $G_{1}$ (Figure 9) and around 


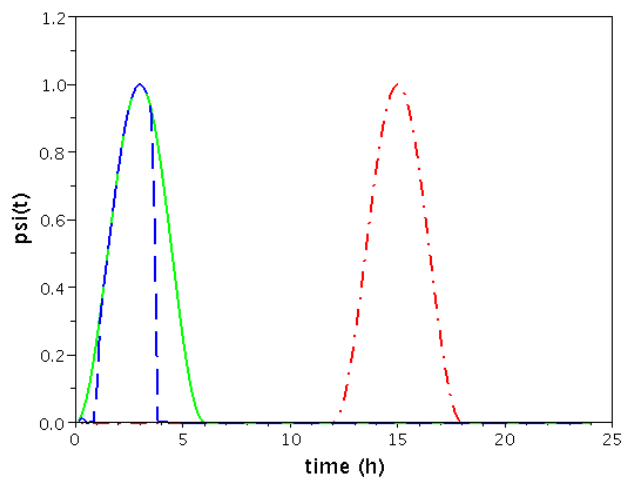

Figure 9: Drug and circadian controls, healthy cell population case. Cosine-like functions modelling the drug and circadian controls for transition from $G_{1}$ to $S / G_{2} / M$ (dash-dotted line) and for transition from $S / G_{2} / M$ to $G_{1}$ in healthy cells. The "natural" (drug-free) control for $S / G_{2} / M$ to $G_{1}$ transition corresponds to the solid line, the drug-induced one to the dashed line.

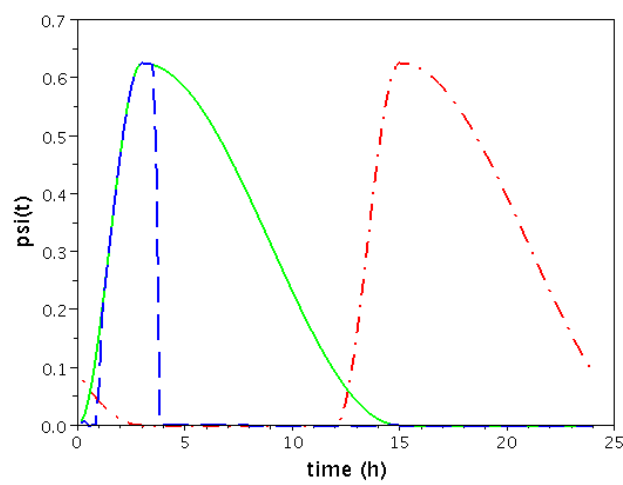

Figure 10: Drug and circadian controls, cancer cell population case. Cosine-like functions modelling the drug and circadian controls for transition from $G_{1}$ to $S / G_{2} / M$ (dash-dotted line) and for transition from $S / G_{2} / M$ to $G_{1}$ in cancer cells. The "natural" (drug-free) control for $S / G_{2} / M$ to $G_{1}$ transition corresponds to the solid line, the drug-induced one to the dashed line. 
3 p.m. for the transition from $G_{1}$ to $S / G_{2} / M$. We assumed that cancer cell populations still obey circadian control at these main checkpoints but, like in [2], we modelled their behaviour by a looser answer to the signal (Figure 10). We assumed that the drug has the same effect on both populations, which couples their behaviours through the drug infusions.

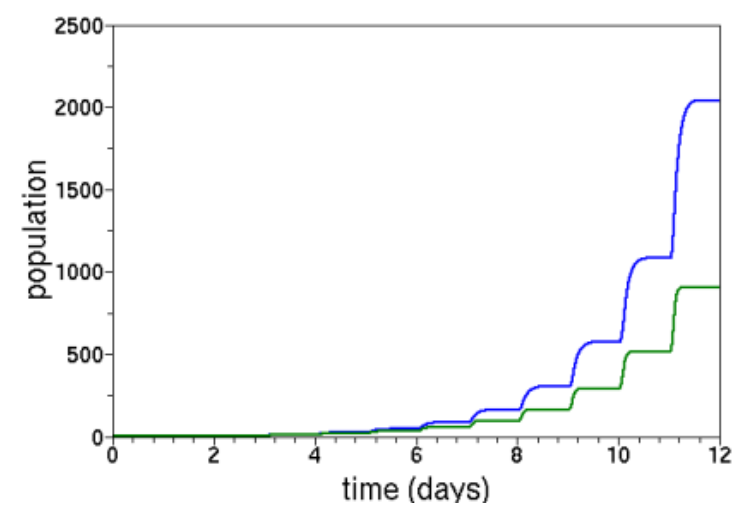

Figure 11: Evolution of the population of cancer (blue, above) and healthy (green, beneath) cells without drug infusion during 12 days. We can see that the populations have different exponential growth rates $\left(\lambda_{\text {cancer }}=0.026\right.$ and $\left.\lambda_{\text {healthy }}=0.024\right)$. Cancer cells proliferate faster than healthy cells.

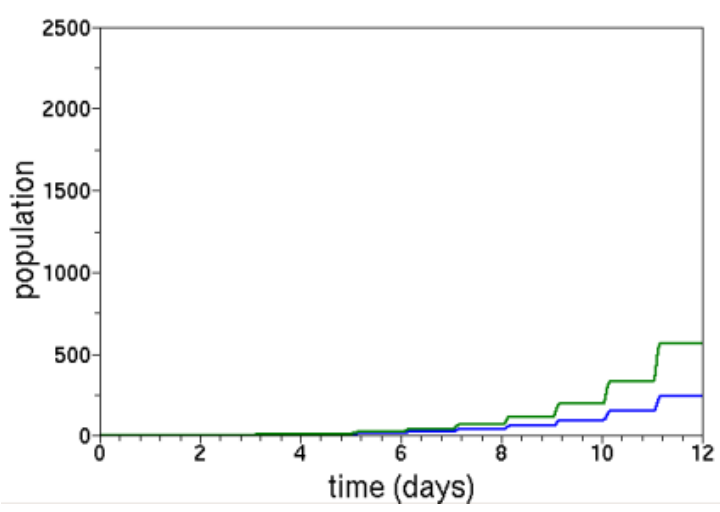

Figure 12: Evolution of the population of cancer (blue, beneath) and healthy (green, above) cells with the drug infusion, starting at time 0 , given by the algorithm. Healthy cells keep multiplying $\left(\lambda_{\text {healthy }}=0.022\right)$ while the cancer cell population is weakened $\left(\lambda_{\text {cancer }}=0.019\right)$. Contrarily to the case without drug, cancer cells proliferate slower than healthy cells.

We solved the constrained optimisation problem with the method of multipliers and the unconstrained minimisations with the gradient method of Section 4.1 . 


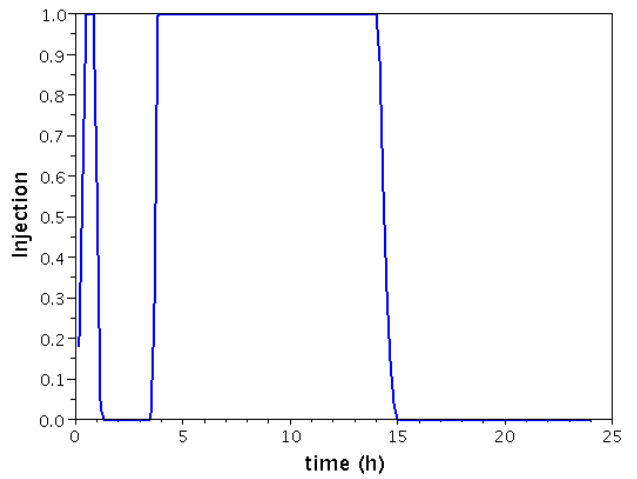

Figure 13: Locally optimal drug infusion strategy (function $g_{2}$, see text for details) found by the optimisation algorithm.

Without drug infusion, the growth rate of cancer cells $\left(0.026 h^{-1}\right)$ is assumed to be larger that the one of healthy cells $\left(0.024 h^{-1}\right)$. This gives an evolution of the respective populations, cancer cells becoming more and more present: see Figure 11. After convergence of the method of multipliers, we get the locally optimal strategy, shown on Figure 13 , defining on $[0 ; 24]$ the $24 \mathrm{~h}$-periodic function $g_{2}$ (recall that $\left.g_{1}=0\right)$.

We can see the action of the locally optimal drug infusion strategy, provided by the optimisation algorithm, on transition rates illustrated on Figures 9 and 10 . This strategy restricts transition time durations from $G_{1}$ to $S / G_{2} / M$ to three hours (between 1 am and $4 \mathrm{am}$ ) for both cell populations, whereas under the drug-free circadian control, transitions would be possible during 6 hours (between midnight and $6 \mathrm{am}$ ) for healthy cells and during 15 hours (between midnight and $3 \mathrm{pm}$ ) for cancer cells. Thus the physical meaning of this locally optimal infusion strategy is to forbid transitions from $G_{1}$ to $S / G_{2} / M$ when cancer cells are under target while healthy cells are not (or very little), thus harming mostly cancer cells. Depending on the toxicity threshold $\Lambda$ chosen, more aggressive drug infusions are possible.

By following the infusion strategy numerically determined by the optimisation algorithm, we obtained that the growth rate of healthy cells was above the chosen toxicity threshold and that the growth rate of cancer cells was strongly weakened. This gave us a description of the evolution of the respective populations, which is illustrated on Figure 12 .

We finally simulated the transition from the stationary state without drug to the stationary state with periodic drug infusion (Figure 14). After a transition of around 10 days, the treatment performs as expected $\left(\lambda_{\text {cancer }}=0.019\right.$ and $\lambda_{\text {healthy }}=0.022$ ). We have thus pulled by this optimal infusion strategy the whole cell population from a state favourable to cancer cells to a state favourable 
to healthy cells.

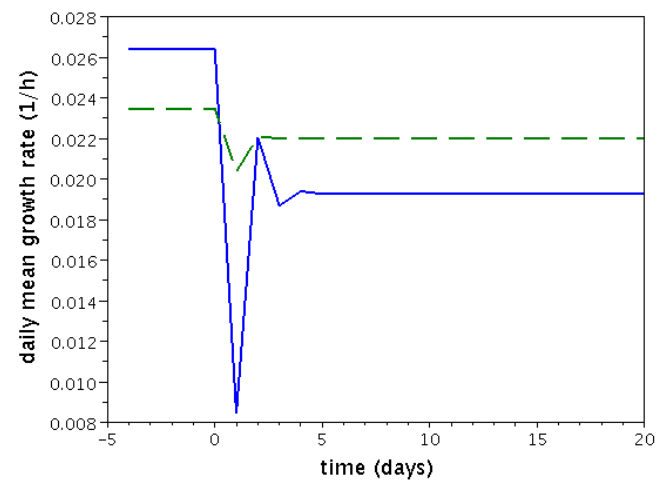

Figure 14: Daily mean growth rates for cancer (solid line) and healthy cells (dashed line) when starting drug infusions at time 0 . After a 10-day transitional phase, the biological system stabilises towards the expected asymptotic growth rate.

\section{Discussion and future prospects for this work}

The problem of circadian control on the cell division cycle in cell populations and its possible applications in clinical oncology is a question of biological and clinical origin that has already been studied from a theoretical point of view in the mathematical setting of age-structured physiological cell population dynamics [31, 32, 33, 34, 35, 36]. Yet many unsolved questions remain, which may be due, in particular, to the scarcity of data on parameters at the individual cell level in proliferating cell populations.

Taking advantage of quantitative measurements obtained by performing recent image analysis techniques of the cell division cycle in individual cells inside a population of non communicating proliferating cells of the same healthy lineage, we have focused in this paper on studying age synchronisation of cells with respect to cell cycle phases. Although these cells are far from an in-vivo situation, they give us precious indications on the age distribution within the cell division cycle in a homogeneous cell population without control exerted on its proliferation, neither by circadian clock messages, nor by applied drugs.

The drug-free experimental proliferation dynamics of this cell population is well approximated by Gamma distributions for cycle phase durations, for which we have shown that the growth exponent $\lambda$, first eigenvalue of the system, is increasing with the variabilities of these durations.

We assumed a multiplicative expression for both temporal controls, physiological (circadian) and pharmacological, onto cell cycle phase age-dependent transition kernels in the McKendrick model of cell proliferation with 2 phases. Our 
results on long-time drug-free cell population dynamics behaviour, as shown on Figures 6 and 8 , are consistent with the theoretical and experimental results presented in [25], that report classical asynchronous cell growth [4, 5, 6, 25, 68, 76], with theoretical works that report entrainment of the phase population densities by periodic control [28], and comparable with the results presented in another modelling context in [1, 2].

We have also been able to propose a new therapeutic optimisation scheme under a toxicity constraint, controlling growth exponents in both cancer and healthy cell populations. We resolved this optimisation problem by using the Uzawa algorithm, which yielded a locally optimal drug infusion strategy.

We can see clear similarities between this infusion strategy and others found in the literature on cancer chronotherapeutic optimisation, although a great variety of models may be considered (see [18] for a comparison of these models). Indeed, in [2, 9] as well as in our case, the suggested infusion schedules set a maximal drug infusion flow when cancer cells get hurt by the drug while healthy cells do not. The modelling settings are however different with respect to the drug effect considered (on death rates in [2, 9, 13] or on proliferation rates, in the present study) and with respect to the model of cell population dynamics chosen. In [9], the solutions (drug delivery flows) to the optimisation problem are much smoother than in the present study. This may be attributed to the fact that here, we represented control by blockade of proliferation (on cell cycle phase transition rates and the resulting growth coefficients), which is closer to a tap open - tap closed' problem than an action on death rates. Whereas most therapeutic control representations so far have been put on death rates (but note that both targets are considered in [51], and comparatively studied in [32, 35, 55]), we know that anticancer drugs act mostly on proliferation, either by damaging the DNA, which results in subsequent cell cycle checkpoint $\left(G_{1} / S\right.$ or $\left.G_{2} / M\right)$ blockade via ATM and p53 in the case of cytotoxics, or by slowing down the $G_{1}$ phase in the case of cytostatics (growth factor inhibitors); this is discussed elsewhere [18]. Drugs that act directly on death rates, e.g., by primarily enhancing the apoptotic cascade may exist (or will exist some day), but are not of common use in the clinic. Hence our choice of the drug target, transition rates, which looks more realistic to us than death rates.

Of note, the authors of [13], also using an age-structured population dynamic model, but with delays, based their optimisation procedure on the remark that the set of 24h-periodic strategies contains both best and worst strategies, depending on the dephasing between the position of the maximal effect of the circadian clock and of the drug infusion. Taking advantage of this remark, they aim at avoiding possible traps (i.e., 'pessimised infusion strategies') when one has no precise idea about the optimal circadian time, thinking that it is advisable to propose a robust optimisation approach by using a different period for the drug infusion scheme. 
Thus, they proposed drug infusion schedules that are not $24 \mathrm{~h}$-periodic, making their drug effects less dependent upon this dephasing (otherwise said, 'shooting more safely in the dark'), which is a quite interesting point of view. In our case, since our approach is based on experimental data supposed to give us insight onto the target and on actual possibilities to reach it safely, we did not consider such a robust optimisation approach.

Our optimisation method relies on the assumption of differences between healthy and cancer cell population model parameters, namely drug-free timedependent circadian control functions $\psi_{i}$ on phase transitions. The clinical feasibility status of the proposed optimal drug infusion strategy is of course still questionable. Indeed, the $\psi_{i}$ functions are thus far purely phenomenological (cosinelike functions) and, further, the local (tissue) drug effect proposed as control variable does not take into account tissue pharmacokinetics-pharmacodynamics (PKPD) of any drug. Last but not least, in view of clinical applications, a wholebody physiologically based PK-PD model is still lacking. Nevertheless, by using this combination of physiologically based modelling of proliferation, mathematical analysis methods, cell imaging and statistical parameter identification techniques, and original optimisation algorithms using eigenvalue control of growth processes, we propose the first steps of a rationale for therapeutic optimisation in oncology at the molecular level in cell populations, healthy and tumour. We intend to complete these first steps in the future, as sketched in [27, 29, 30], to get closer to the clinic.

Various measurements needed to identify parameters of our model control functions were still out of reach by the biological experiments performed in this pioneering study, and this is the reason why we used only a phenomenological representation (by plain cosines) of circadian control, but more measurements are expected to come from further experiments performed by biologists on samples of cell populations, healthy or tumour, with or without circadian control.

In particular, in forthcoming recordings of FUCCI data on healthy and cancer cells, we will pay attention to parallel experimental measurements of the growth exponents (that are inverses of doubling times multiplied by a factor $\ln 2$ ) in the proliferating cell populations at stake. We will thus identify experimentally the growth coefficient $\lambda$ on cell population samples, in which we will simultaneously identify the parameters of our model from cell cycle phase duration distributions by FUCCI recordings, obtaining a theoretical growth exponent by solving the Euler-Lotka equation. This will allow us to validate (or falsify) the model. But thus far, such simultaneous measurements have not been made available to us.

In the immediate future, we intend firstly to make use of a 3-phase, rather than 2-phase, model. In the absence of fluorescent markers for the $G_{2} / M$ transition thus far, we will investigate the possibility that $S / G_{2} / M$ variability may in fact be sheer $S / G_{2}$ variability, neglecting $M$ phase variability, that is indeed very short, 
and for which transition to $G_{1}$ phase might be modelled by a Dirac-like probability measure (i.e., fixed duration of $M$ phase). This hypothesis still needs to be documented, but if it is valid, then our methodology will give us access to both $G_{1} / S$ and $G_{2} / M$ transitions, that are the most important ones for both circadian and pharmacological control.

Secondly, with more FUCCI data on both cancer and healthy cell populations, we should be able to compare synchronisation and control in different settings in these two situations, as much as possible in cell populations with the same histological origin. In this way, using the methodology described in this study, we should be able to assess the assumptions mentioned in Section 1, examining the part played by circadian clocks in synchronisation of cells in a given population, and to use new observations to propose new therapeutic tracks in oncology, more precisely in cancer chronotherapeutics.

\section{Acknowledgments}

This work has been supported by a grant from the European Research Area in Systems Biology (ERASysBio+) to the French National Research Agency (ANR) \#ANR-09-SYSB-002-004 for the research network Circadian and Cell Cycle Clock Systems in Cancer (C5Sys) coordinated by Francis Lévi (Villejuif, France).

The authors heartfully thank G. van der Horst and F. Tamanini at Erasmus University, Rotterdam, for providing us in the course of scientific exchanges within the C5Sys network with FUCCI data carefully defined, constituted and analysed by one of us (S. Saito) in G. van der Horst's lab at Erasmus University.

\section{References}

[1] A. Altinok, D. Gonze, F. Lévi, A. Goldbeter, An automaton model for the cell cycle, Interface focus 1 (2011) 36-47.

[2] A. Altinok, F. Lévi, A. Goldbeter, A cell cycle automaton model for probing circadian patterns of anticancer drug delivery, Adv. Drug Deliv. Rev. 59 (2007) 1036-1010.

[3] A. Altinok, F. Lévi, A. Goldbeter, Identifying mechanisms of chronotolerance and chronoefficacy for the anticancer drugs 5-fluorouracil and oxaliplatin by computational modeling, Eur. J. Pharm. Sci. 36 (2009) 20-38.

[4] O. Arino, A survey of structured cell population dynamics, Acta Biotheor. 43 (1995) 3-25.

[5] O. Arino, M. Kimmel, Comparison of approaches to modeling of cell population dynamics, SIAM J. Appl. Math. 53 (1993) 1480-1504. 
[6] O. Arino, E. Sanchez, A survey of cell population dynamics, J. Theor. Med. 1 (1997) 35-51.

[7] M.J. Ausserlechner, P. Obexer, G. Böck, S. Geley, R. Kofler, Cyclin D3 and c-MYC control glucocorticoid-induced cell cycle arrest but not apoptosis in lymphoblastic leukemia cells, Cell death differ. 11 (2004) 165-174.

[8] N. Baghdassarian, R. Catallo, M.A. Mahly, P. Ffrench, F. Chizat, P.A. Bryon, M. Ffrench, Glucocorticoids induce G1 as well as S-phase lengthening in normal human stimulated lymphocytes: differential effects on cell cycle regulatory proteins, Exp. Cell Res. 240 (1998) 263-273.

[9] C. Basdevant, J. Clairambault, F. Lévi, Optimisation of time-scheduled regimen for anti-cancer drug infusion, Mathematical Modelling and Numerical Analysis 39 (2006) 1069-1086.

[10] F. Bekkal Brikci, J. Clairambault, B. Perthame, Analysis of a molecular structured population model with polynomial growth for the cell cycle, Mathematical and Computer Modelling 47 (2008) 699-713.

[11] F. Bekkal Brikci, J. Clairambault, B. Ribba, B. Perthame, An age-andcyclin-structured cell population model for healthy and tumoral tissues, Journal of Mathematical Biology 57 (2008) 91-110.

[12] A. Berman, R.J. Plemmons, Nonnegative matrices in the mathematical sciences, Amer. Math. Soc., 1994.

[13] S. Bernard, B. Čajavec Bernard, F. Lévi, H. Herzel, Tumor growth rate determines the timing of optimal chronomodulated treatment schedules, PLoS Computational Biology 6(3) (2010) e1000712.

[14] S. Bernard, L. Pujo-Menjouet, M.C. Mackey, Analysis of cell kinetics using a cell division marker: Mathematical modeling of experimental data, Biophys. J. 84 (2003) 3414-3424.

[15] D.P. Bertsekas, On the Goldstein - Levitin - Polyak gradient projection method, IEEE transactions on automatic control AC-21 (1976) 174-184.

[16] D.P. Bertsekas, Constrained Optimization and Lagrange multiplier method, Academic Press, NY; republished by Athena Scientific, MA,1997, 1982.

[17] D.P. Bertsekas, Nonlinear Programming, Athena Scientific, 1995. 
[18] F. Billy, J. Clairambault, O. Fercoq, Optimisation of cancer drug treatments using cell population dynamics, in: U. Ledzewicz, H. Schättler, A. Friedman, E. Kashdan (Eds.), Mathematical Methods and Models in Biomedicine, Springer, 2012, pp. 263-299. In press.

[19] G.A. Bjarnason, R.C.K. Jordan, R.B. Sothern, Circadian variation in the expression of cell-cycle proteins in the human oral epithelium, Am. J. Pathol 154 (1999) 613-622.

[20] G.A. Bjarnason, R.C.K. Jordan, P.A. Wood, Q. Li, D.W. Lincoln, R.B. Sothern, W.J. Hrushesky, Y. Ben-David, Circadian expression of clock genes in human oral mucosa and skin: association with specific cell-cycle phases, Am. J. Pathol 158 (2001) 1793-1801.

[21] G. Bocci, R. Danesi, A.D. Di Paolo, F. Innocenti, G. Allegrini, A. Falcone et al., Comparative pharmacokinetic analysis of 5-fluorouracil and its major metabolite 5- fluoro-5,6-dihydrouracil after conventional and reduced test dose in cancer patients, Clin. Cancer Res. 6 (2000) 3032-37.

[22] J.F. Bonnans, J.C. Gilbert, C. Lemaréchal, C. Sagastizábal, Numerical Optimization - Theoretical and Practical Aspects, Universitext, Springer Verlag, Berlin, 2006.

[23] R.M. Buijs, A. Kalsbeek, Hypothalamic integration of central and peripheral clocks, Nature Rev. Neurosci. 2 (2001) 521-526.

[24] F.J. Burns, I.F. Tannock, On the existence of a $G_{0}$ phase in the cell cycle, Cell Tissue Kinet. 3 (1970) 321-334.

[25] G. Chiorino, J.A.J. Metz, D. Tomasoni, P. Ubezio, Desynchronization rate in cell populations: mathematical modeling and experimental data, J. Theor. Biol. 208 (2001) 185-199.

[26] J. Cicenas, M. Valius, The cdk inhibitors in cancer research and therapy, J. Cancer Res. Clin. Oncol. 137 (2011) 1409-1418.

[27] J. Clairambault, Modelling oxaliplatin drug delivery to circadian rhythm in drug metabolism and host tolerance, Adv. Drug Deliv. Rev. 59 (2007) 10541068 .

[28] J. Clairambault, A step toward optimization of cancer therapeutics. physiologically based modelling of circadian control on cell proliferation, IEEEEMB Magazine 27 (2008) 20-24. 
[29] J. Clairambault, Modelling physiological and pharmacological control on cell proliferation to optimise cancer treatments, Mathematical Modelling of Natural Phenomena 4 (2009) 12-67.

[30] J. Clairambault, Optimising cancer pharmacotherapeutics using mathematical modelling and a systems biology approach, Personalized Medicine 8 (2011) 271-286.

[31] J. Clairambault, S. Gaubert, T. Lepoutre, Comparison of Perron and Floquet eigenvalues in age structured cell division models, Mathematical Modelling of Natural Phenomena 4 (2009) 183-209.

[32] J. Clairambault, S. Gaubert, T. Lepoutre, Circadian rhythm and cell population growth, Mathematical and Computer Modelling 53 (2011) 1558-1567.

[33] J. Clairambault, S. Gaubert, B. Perthame, An inequality for the Perron and Floquet eigenvalues of monotone differential systems and age-structured equations, C. R. Acad. Sci. (Paris) Ser. I Mathématique 345 (2007) 549554.

[34] J. Clairambault, B. Laroche, S. Mischler, B. Perthame, A mathematical model of the cell cycle and its control, Technical Report, INRIA, Domaine de Voluceau - Rocquencourt - BP 105 - 78153 Le Chesnay Cedex (France), 2003.

[35] J. Clairambault, P. Michel, B. Perthame, Circadian rhythm and tumour growth, C. R. Acad. Sci. (Paris) Ser. I Mathématique (Équations aux dérivées partielles) 342 (2006) 17-22.

[36] J. Clairambault, P. Michel, B. Perthame, A model of the cell cycle and its circadian control, in: A. Deutsch, L. Brusch, H. Byrne, G. de Vries, J. Herzel (Eds.), Mathematical Modeling of Biological Systems, Volume I: Cellular Biophysics, Regulatory Networks, Development, Biomedicine, and Data Analysis, Birkhäuser, Boston, 2007, pp. 239-251.

[37] A.A. Cohen, T. Kalisky, A. Mayo, N. Geva-Zatorsky, T. Danon, I. Issaeva, R. Kopito, N. Perzov, R. Milo, A. Sigal, U. Alon, Protein dynamics in individual human cells: Experiment and theory, PLoS one 4 (2009) 1-12. E4901.

[38] R.B. Diasio, B.E. Harris, Clinical pharmacology of 5-fluorouracil, Clin. Pharmacokinet. 16 (1989) 215-237.

[39] E. Filipski, P.F. Innominato, M.W. Wu, X.M. Li, S. Iacobelli, L.J. Xian, F. Lévi, Effect of light and food schedules on liver and tumor molecular clocks in mice, J. Natl. Cancer Inst. 97 (2005) 507-517. 
[40] E. Filipski, V.M. King, X.M. Li, T.G. Granda, F. Lévi, Host circadian clock as a control point in tumor progression, J. Natl. Cancer Inst. 94 (2002) 690697.

[41] L. Fu, H. Pelicano, J. Liu, P. Huang, C.C. Lee., The circadian gene per2 plays an important role in tumor suppression and DNA damage response in vivo, Cell 111 (2002) 41-50.

[42] S.P. Garbett, P. Gabriel, D.R. Tyson, G.F. Webb, The contribution of age structure to cell population responses to targeted therapeutics, 2011. Submitted.

[43] J.H. Goldie, A.J. Coldman, Drug resistance in cancer: mechanisms and models, Cambridge University Press, 1998.

[44] A.A. Goldstein, Convex programming in hilbert space, Bull. Amer. Math. Soc. 70 (1964) 709-710.

[45] A.A. Goldstein, Constructive Real Analysis, Harper \& Row, New York, 1967.

[46] G.H. Golub, C.F. Van Loan, Matrix computations, Johns Hopkins University Press, Baltimore, Md, 1989.

[47] A. Gréchez-Cassiau, B. Rayet, F. Guillaumond, M. Teboul, F. Delaunay, The circadian clock component bmal1 is a critical regulator of p21(waf1/cip1) expression and hepatocyte proliferation, J. Biol. Chem. 283 (2008) 453542.

[48] M. Gyllenberg, A. Osipov, L. Päivärinta, The inverse problem of linear agestructured population dynamics, J. Evol. Equations 2 (2002) 223-239.

[49] M.B. Kastan, J. Bartek, Cell-cycle checkpoints and cancer, Nature 432 (2004) 316-323.

[50] T. Kato, Perturbation Theory for Linear Operators, Springer-Verlag Berlin and Heidelberg GmbH \& Co. K, 1966.

[51] M. Kimmel, A. Świerniak, Control theory approach to cancer chemotherapy: Benefiting from phase dependence and overcoming drug resistance, in: A. Friedman (Ed.), Tutorials in Mathematical Biosciences III, volume 1872 of Lecture Notes in Mathematics, Springer Berlin / Heidelberg, 2006, pp. $185-221$. 
[52] N. Komarova, D. Wodarz, Drug resistance in cancer: principles of emergence and prevention, Proc. Natl. Acad. Sci. U S A 102 (2005) 9714-9719.

[53] R.K. Konopka, S. Benzer, Clock mutants of drosophila melanogaster, Proc. Natl. Acad. Sci. U S A 68 (1971) 2112-16.

[54] A. Kramer, F. Yang, P. Snodgrass, X. Li, T.E. Scammell, Regulation of daily locomotor activity and sleep by hypothalamic EGF receptor signaling, Science 294 (2001) 2511-15.

[55] T. Lepoutre, Analyse et modélisation de phénomènes de croissance et mouvement issus de la biologie, Ph.D. thesis, Université Paris VI Pierre et Marie Curie, 4, place Jussieu - 75005 Paris (France), 2009. Available online from http://tel.archives-ouvertes.fr/tel-00457561/fr/.

[56] F. Lévi, Cancer chronotherapeutics, Special issue of Chronobiology International 19 (2002) 1-19.

[57] F. Lévi, Chronotherapeutics: the relevance of timing in cancer therapy, Cancer Causes Control 17 (2006) 611-621.

[58] F. Lévi, The circadian timing system: A coordinator of life processes. implications for the rhythmic delivery of cancer therapeutics, IEEE-EMB Magazine 27 (2008) 17-20.

[59] F. Lévi, A. Altinok, J. Clairambault, A. Goldbeter, Implications of circadian clocks for the rhythmic delivery of cancer therapeutics, Phil. Trans. Roy. Soc. A 366 (2008) 3575-3598.

[60] F. Lévi, A. Okyar, S. Dulong, P.F. Innominato, J. Clairambault, Circadian timing in cancer treatments, Ann. Rev. Pharmacol. Toxicol. 50 (2010) 377421.

[61] F. Lévi, U. Schibler, Circadian rhythms: Mechanisms and therapeutic implications, Ann. Rev. Pharmacol. Toxicol. 47 (2007) 493-528.

[62] A.S. Lewis, M.L. Overton, Eigenvalue optimization, Acta Numerica 5 (1996) 149-190.

[63] H. Lodish, A. Berk, C.A. Kaiser, M. Krieger, M.P. Scott, A. Bretscher, H. Ploegh, P.T. Matsudaira, Molecular Cell Biology, W. H. Freeman, New York, 6th edition, 2007. 
[64] L. Ma, J. Wagner, J.J. Rice, W. Hu, A.J. Levine, G.A. Stolovitzky, A plausible model for the digital response of p53 to DNA damage, Proc. Natl. Acad. Sci. 102 (2005) 14266-71.

[65] T. Matsuo, S. Yamaguchi, S. Mitsuia, A. Emi, F. Shimoda, H. Okamura, Control mechanism of the circadian clock for timing of cell division in vivo, Science 302 (2003) 255-259.

[66] H.H. McAdams, A. Arkin, Stochastic mechanisms in gene expression, Proc. Natl. Acad. Sci. USA 31 (1997) 814-819.

[67] A.G. McKendrick, Applications of mathematics to medical problems, Proc. Edinburgh Math. Soc. 54 (1926) 98-130.

[68] J.A.J. Metz, O. Diekmann, The dynamics of physiologically structured populations, volume 68 of Lecture notes in biomathematics, Springer, New York, 1986.

[69] P. Michel, S. Mischler, B. Perthame, General entropy equations for structured population models and scattering, C. R. Acad. Sci. (Paris) Ser. I Mathématique 338 (2004) 697-702.

[70] P. Michel, S. Mischler, B. Perthame, The entropy structure of models of structured population dynamics. General Relative Entropy inequality: an illustration on growth models, J. Math. Pures et Appl. 84 (2005) 1235-1260.

[71] D. Morgan, The Cell Cycle: Principles of Control, Primers in Biology series, Oxford University Press, 2006.

[72] M.C. Mormont, J. Waterhouse, P. Bleuzen, S. Giacchetti, A. ami, A. Bogdan, J. Lellouch, J.L. Misset, Y. Touitou, F. Lévi, Marked 24-h rest-activity rhythms are associated with better quality of life, better response and longer survival in patients with metastatic colorectal cancer and good performance status, Clin. Cancer Res. (2000) 3038-3045.

[73] J. Nocedal, S. Wright, Numerical Optimization, Springer, New York, 1999.

[74] M.L. Overton, Large-scale optimization of eigenvalues, SIAM Journal on Optimization 2 (1991) 88-120.

[75] M.L. Overton, R.S. Womersley, On minimizing the spectral radius of a nonsymmetric matrix function: optimality conditions and duality theory, SIAM Journal on Matrix Analysis and Applications 9 (1988) 473-498. 
[76] B. Perthame, Transport Equations in Biology, Frontiers in Mathematics series, Birkhäuser, Boston, 2007.

[77] G.J. Peters, J. Lankelma, R.M. Kok, P. Noordhuis, C.J. van Groeningen, C.L. van der Wilt etal., Prolonged retention of high concentrations of 5fluorouracil in human and murine tumors as compared with plasma, Cancer Chemother. Pharmacol. 31 (1993) 269-276.

[78] S.M. Reppert, D.R. Weaver, Coordination of circadian timing in mammals, Nature 418 (2002) 935-941.

[79] T. Rich, P.F. Innominato, J. Boerner, M.C. Mormont, S. Iacobelli, B. Baron, C. Jasmin, F. Lévi, Elevated serum cytokines correlated with altered behavior, serum cortisol rhythm, and dampened 24-hour rest-activity pattern in patients with metastatic colorectal cancer, Clin. Cancer Res. 11 (2005) 1757-64.

[80] A. Sakaue-Sawano, H. Kurokawa, T. Morimura, A. Hanyu, H. Hama, H. Osawa, S. Kashiwagi, K. Fukami, T. Miyata, H. Miyoshi, T. Imamura, M. Ogawa, H. Masai, A. Miyawaki, Visualizing spatiotemporal dynamics of multicellular cell-cycle progression, Cell 32 (2008) 487-498.

[81] A. Sakaue-Sawano, K. Ohtawa, H. Hama, M. Kawano, M. Ogawa, A. Miyawaki, Tracing the silhouette of individual cells in $\mathrm{S} / \mathrm{G} 2 / \mathrm{M}$ phases with fluorescence, Chem Biol. 15 (2008) 1243-48.

[82] U. Schibler, P. Sassone-Corsi, A web of circadian pacemakers, Cell 111 (2002) 919-922.

[83] V. Shahreazaei, P.G. Swain, Analytical distributions for stochastic gene expression, Proc. Natl. Acad. Sci. USA 105 (2008) 17256-17261.

[84] E. Sherer, E. Tocce, R.E. Hannemann, A.E. Rundell, D. Ramkrishna, Identification of age-structured models: cell cycle phase transitions, Biotechnol. Bioeng. 99 (2008) 960-974.

[85] R. Smaaland, O.D. Laerum, K. Lote, O. Sletvold, R.B. Sothern, R. Bjerknes, DNA synthesis in human bone marrow is circadian stage dependent, Blood 77 (1991) 2603-2611.

[86] R.G. Staudte, Planning blocked mitosis experiments for efficient estimation of population-doubling time and cell-cycle time, Biometrics 38 (1982) 777785. 
[87] K. Vermeulen, D.R. Van Bockstaele, Z.N. Berneman, The cell cycle: a review of regulation, deregulation and therapeutic targets in cancer, Cell Prolif. 36 (2003) 131-149.

[88] M.H. Vitaterna, D.P. King, A.M. Chang, J.M. Kornhauser, P.L. Lowrey, J.D. McDonald, W.F. Dove, L.H. Pinto, F.W. Turek, J.S. Takahashi, Mutagenesis and mapping of a mouse gene, clock, essential for circadian behavior, Science 264 (1994) 719-725.

[89] B. Vogelstein, D. Lane, A.J. Levine, Surfing the p53 network, Nature 408 (2000) 307-310.

[90] T. Zhang, P. Brazhnik, J.J. Tyson, Exploring mechanisms of the DNAdamage response: p53 pulses and their possible relevance to apoptosis, Cell Cycle (2007) 85-94.

[91] X. Zhang, F. Liu, W.Wang, Two-phase dynamics of p53 in the DNA damage response, Proc. Natl. Acad. Sci. USA (2011). Epub ahead of print.

[92] L. Zitvogel, L. Apetoh, F. Ghiringhelli, G. Kroemer, Immunological aspects of cancer chemotherapy, Nature Rev. Immunol. 8 (2008) 59-73. 